\title{
RESEARCH
}

Open Access

\section{The osteogenic differentiation of human adipose-derived stem cells is regulated through the let-7i-3p/LEF1/ $\beta$-catenin axis under cyclic strain}

Yadong Luo ${ }^{1,2+}$, Ran Ge ${ }^{3 \dagger}$, Heming $\mathrm{Wu}^{1,2+}$, Xu Ding ${ }^{1,2}$, Haiyang Song ${ }^{1,2}$, Huan Ji ${ }^{1,2}$, Meng Li ${ }^{1,2}$, Yunan Ma ${ }^{1,2}$, Sheng $\mathrm{Li}^{1,2}$, Chenxing Wang ${ }^{1,2}$ and Hongming $\mathrm{Du}^{1,2^{*}}$ (D)

\begin{abstract}
Background: The Wnt/B-catenin pathway is involved in the osteogenic differentiation of human adipose-derived stem cells (hASCs) under cyclic strain. Very little is known about the role of microRNAs in these events.

Methods: Cells were obtained using enzyme digestion methods, and proliferation was detected using Cell Counting Kit 8. Cell cycles and immunophenotypes were detected by flow cytometry. The multilineage potential of hASCs was induced by induction media. Cyclic strain was applied to hASCs $(0.5 \mathrm{~Hz}, 2 \mathrm{~h} /$ day, 6 days) to induce osteogenic differentiation and miRNA changes. Bioinformatic and dual-luciferase analyses confirmed lymphoid enhancer factor 1 (LEF1) as a potential target of let-7i-3p. The effect of let-7i-3p on LEF1 in hASCs transfected with a let-7i-3p mimic and inhibitor was analyzed by immunofluorescence. hASCs were transfected with a let-7i-3p mimic, inhibitor, or small interfering RNA (siRNA) against LEF1 and $\beta$-catenin. Quantitative real-time PCR (qPCR) and western blotting were performed to examine the osteogenic markers and Wnt/B-catenin pathway at the mRNA and protein levels, respectively. Immunofluorescence and western blotting were performed to confirm the activation of the $\mathrm{Wnt} / \beta$-catenin pathway.

Results: Flow cytometry showed that $82.12 \% \pm 5.83 \%$ of the cells were in G1 phase and $17.88 \% \pm 2.59 \%$ of the cells were in S/G2 phase; hASCs were positive for CD29, CD90, and CD105. hASCs could have the potential for osteogenic, chondrogenic, and adipogenic differentiation. MicroRNA screening via microarray showed that let-7i-3p expression was decreased under cyclic strain. Bioinformatic and dual-luciferase analyses confirmed that LEF1 in the Wnt/B-catenin pathway was the target of let-7i-3p. Under cyclic strain, the osteogenic differentiation of hASCs was promoted by overexpression of LEF1and $\beta$-catenin and inhibited by overexpression of let-7i-3p. hASCs were transfected with let-7i-3p mimics and inhibitor. Gain- or loss-of-function analyses of let-7i-3p showed that the osteogenic differentiation of hASCs was promoted by decreased let-7i-3p expression and inhibited by increased let-7i-3p expression. Furthermore, high LEF1 expression inactivated the Wnt/ $\beta$-catenin pathway in let-7i-3p-enhanced hASCs. In contrast, let-7i-3p inhibition activated the Wnt/ß-catenin pathway.
\end{abstract}

Conclusions: Let-7i-3p, acting as a negative regulator of the Wnt/B-catenin pathway by targeting LEF1, inhibits the osteogenic differentiation of hASCs under cyclic strain in vitro.

Keywords: hASC osteogenic differentiation, Cyclic strain, Wnt/ß-catenin pathway, Let-7i-3p, LEF1

\footnotetext{
* Correspondence: dhm_010@sina.com

${ }^{\dagger}$ Yadong Luo, Ran Ge and Heming Wu contributed equally to this work.

'Department of Oral and Maxillofacial Surgery, Affiliated Stomatological Hospital of Nanjing Medical University, Hanzhong Road No.136, Nanjing 210029, Jiangsu Province, People's Republic of China

${ }^{2}$ Oral Disease Key Laboratory of Jiangsu Province, Nanjing Medical University,

Nanjing 210029, Jiangsu Province, People's Republic of China

Full list of author information is available at the end of the article
}

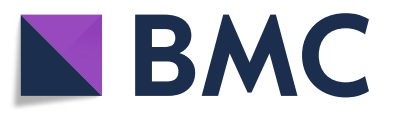

(c) The Author(s). 2019 Open Access This article is distributed under the terms of the Creative Commons Attribution 4.0 International License (http://creativecommons.org/licenses/by/4.0/), which permits unrestricted use, distribution, and reproduction in any medium, provided you give appropriate credit to the original author(s) and the source, provide a link to the Creative Commons license, and indicate if changes were made. The Creative Commons Public Domain Dedication waiver (http://creativecommons.org/publicdomain/zero/1.0/) applies to the data made available in this article, unless otherwise stated. 


\section{Introduction}

Bone tissue engineering can be used to repair bone defects induced by various factors by construction of functional bone tissue in vitro [1]. Seed cells, bioscaffold materials, and cell and bioscaffold materials in combination are the three significant components of bone tissue engineering [2]. Among them, research on seed cells is one focus of the bone tissue engineering field [3].

Bone marrow mesenchymal stem cells (BMSCs) are currently the most widely used seed cells in the field of bone tissue engineering [4]. However, there are still some problems in the practical application of BMSCs, such as limited cell acquisition, complex cellular components, and decreased osteogenic differentiation in aging cell donors [5]. In light of this, researchers began looking for other types of adult stem cells as seed cells for bone tissue engineering. In 2001, Zuk et al. first obtained human adipose-derived stem cells (hASCs), which have multilineage differentiation potential, including osteogenic, chondrogenic, and adipogenic differentiation abilities [6]. The advantages of ASCs over BMSCs include abundant cell sources, easy access, good expansion, and stable osteogenic differentiation activity in vitro [7, 8]. While numerous papers also describe the disadvantages of ASC vs BMSC osteogenesis [9], many scholars still believe that ASCs are a valuable new seed cell source in bone tissue engineering $[10,11]$.

How to promote the osteogenic differentiation of ASCs in vitro has become an important research area for bone tissue engineering. Mechanical loading plays an essential role in cellular biological responses, such as the regulation of protein synthesis, cell proliferation, and differentiation [12]. Some studies have shown that tensile stress can promote the osteogenic differentiation of adult MSCs, including hASCs [13]. The above results suggest that tensile stress stimulation can promote the osteogenic differentiation of ASCs.

The Wnt signaling pathway is involved in the regulation of many critical biological processes, including embryogenesis and development, stem cell maintenance and differentiation, energy metabolism balance, and tissue stability maintenance. The Wnt/ $\beta$-catenin pathway plays a complicated role in bone biology. Some syndromes show low bone mass and, consequently, frequent bone fractures caused by loss of functional mutations of the coreceptor LRP5 [14], while gain-of-function mutations of the LRP5 receptor lead to high bone mass [15]. Bone thickness was shown to be decreased in mice Wnt16 knockout mice [16]. In contrast, LEF1 was shown to be overexpressed in the initial and later phases of bone repair [17]. The activated $\mathrm{Wnt} / \beta$-catenin pathway can promote the osteogenic differentiation of BMSCs [18]. Although the activation of the Wnt/ $\beta$-catenin pathway results in bone formation, increased bone mass results from excessive bone formation rather than bone resorption in both situations [19]. Previous studies have also found that tensile stress activates the Wnt/ $\beta$-catenin pathway, which promotes osteogenic differentiation [20, 21].

MicroRNAs (miRNAs) are highly conserved singlestranded noncoding RNA molecules consisting of 18-22 nucleotides. MiRNAs bind to the 3'UTRs of their target mRNAs, inhibiting their translation or promoting their degradation [22]. miRNAs are involved in complex biological processes in organisms, including cell division, differentiation, apoptosis, and organ development [22]. Notably, miRNAs are involved in regulating the osteogenic differentiation of adult MSCs by regulating the expression of Wnt/ $\beta$-catenin pathway molecules. For example, miR-335-5p activates the $\mathrm{Wnt} / \beta$-catenin pathway by inhibiting Dkk1 expression, thereby promoting osteogenic differentiation [23]. miR-138 and miR-30e regulate the expression of FAK and LRP6, respectively, thereby inhibiting the osteogenic differentiation of MSCs $[24,25]$. Although existing research results indicate that miRNAs play an essential role in cell osteogenic differentiation of cells by regulating the $\mathrm{Wnt} / \beta$-catenin pathway, research in this field is still incomplete due to the diversity of miRNAs and their target genes, and additional studies should be performed.

In this study, a gene microarray was used to screen the altered expression levels of miRNAs in hASCs during osteogenic differentiation under cyclic strain. We analyzed miRNAs associated with $\mathrm{Wnt} / \beta$-catenin by bioinformatics methods and found that the target gene regulated by hsa-let-7i-3p is most likely lymphoid enhancer factor 1 (LEF1) in the $\mathrm{Wnt} / \beta$-catenin pathway. We hypothesize that cyclic strain activates the Wnt/ $\beta$ catenin pathway by downregulating hsa-let-7i-3p, thereby promoting the osteogenic differentiation of hASCs. This study aimed to validate this hypothesis using cell and molecular biology methods.

\section{Materials \\ Cell culture}

Fat tissue was obtained from 6 donors who underwent flap reconstruction in the Oral and Maxillofacial Surgery Department at the Affiliated Stomatological Hospital of Nanjing Medical University, and all patients signed informed consent. The procedures were approved by the ethics committee of Nanjing Medical University. Adipose tissue was isolated from flaps and then washed three times with phosphate-buffered saline (PBS) to remove red blood cells. The adipose tissue was digested with $0.1 \%$ collagenase type I (BD, USA) at $37^{\circ} \mathrm{C}$ for $1 \mathrm{~h}$. After tissue digestion, the tissue was centrifuged at $300 \mathrm{~g}$ for $5 \mathrm{~min}$, after which the supernatant was discarded, and the pellet was resuspended twice. Resuspend cells 
were cultured in alpha-modified Eagle's medium ( $\alpha$ MEM, Gibco BRL, USA) containing 10\% fetal bovine serum (FBS; Gibco BRL, USA) at $37^{\circ} \mathrm{C}$ in a $5 \% \mathrm{CO}_{2}$ incubator. In this study, fourth-passage cells were used in the following experiments.

\section{Cell proliferation}

Cell proliferation was detected following the manufacturer's manual by Cell Counting Kit- 8 assays (CCK- 8 , Beyotime, China) according to the manufacturer's instructions. Cells were seeded into 96-well plates at 3000 cells per well and then cultured in $\alpha$-MEM supplemented with $10 \% \mathrm{FBS}$ at $37^{\circ} \mathrm{C}$ in a $5 \% \mathrm{CO}_{2}$ incubator. Cells in 6 wells were measured by adding $10 \mu \mathrm{l}$ of Cell Counting Kit-8 solution to each well, followed by incubation at $37^{\circ} \mathrm{C}$ for $2 \mathrm{~h}$. Absorbance was measured by spectrophotometry (SPECTRA MAX190, Sunnyvale, CA, USA) at $450 \mathrm{~nm}$. Cell counting was performed for 8 consecutive days. The growth curve of the cells was drawn according to the above results.

\section{Cell cycle and immunophenotype detection by flow cytometry}

Cells were collected by centrifugation and fixed with precooled $70 \%$ ethanol overnight. Cells were added to $50 \mu \mathrm{L}$ of RNase stock solution $(100 \mu \mathrm{g} / \mathrm{mL})$, stained with $500 \mu \mathrm{L}$ of PBS containing $50 \mu \mathrm{g} / \mathrm{mL}$ ethidium bromide (PI), and incubated at $4{ }^{\circ} \mathrm{C}$ for $30 \mathrm{~min}$ in the dark. Finally, the fluorescence intensity of DNA-PI was detected by a FACSVerse flow cytometer (BD, USA) for analysis of cell cycle changes.

Immunophenotyping of the hASCs was performed by staining for $30 \mathrm{~min}$ on ice in the dark with the fluorochrome-labeled monoclonal antibodies CD29-APC (BD, USA), CD34-PE (BD, USA), CD45-PE (BD, USA), CD90-FITC (BD, USA), and CD105-PerCP cy5.5 (BD, USA). Untreated cells served as the negative control to adjust for the compensation of fluorochrome overlap. The fluorescence intensity was examined by a FACSVerse flow cytometer (BD, USA), and flow cytometry analysis was performed with FlowJo software 7.6.1 (Leonard Herzenberg, USA).

\section{Multilineage potential of hASCs}

hASCs were induced toward adipogenic, osteogenic, and chondrogenic differentiation to confirm their multilineage potential. Adipogenic medium (10\% FBS, $1 \mu \mathrm{M}$ dexamethasone, $200 \mu \mathrm{M}$ indomethacin, $10 \mathrm{mg} / \mathrm{L}$ insulin, and $0.5 \mathrm{mM} 3$-isobutyl-1-methylxanthine in $\alpha$-MEM) was used to induce adipogenic differentiation of hASCs; after 14 days, hASCs were stained with Oil Red O to determine the formation of lipid droplets. Osteogenic medium (10\% FBS, $0.1 \mu \mathrm{M}$ dexamethasone, $10 \mathrm{mM} \beta$-glycerol phosphate, and $50 \mu \mathrm{M}$ vitamin $\mathrm{C}$ in $\alpha$-MEM) was used to induce osteogenic differentiation of hASCs; 21 days later, hASCs were stained with Alizarin Red to confirm the existence of mineralized nodules. Chondrogenic medium (10\% FBS, $10 \mathrm{ng} / \mathrm{ml}$ TGF- $\beta 1,200 \mu \mathrm{M}$ indomethacin, $6.25 \mu \mathrm{g} / \mathrm{ml}$ insulin, and $50 \mathrm{nM}$ vitamin $\mathrm{C}$ in $\alpha$-MEM) was used to induce chondrogenic differentiation of hASCs; 14 days later, hASCs were stained with Alcian Blue to verify the existence of proteoglycans.

\section{Cyclic strain loading on hASCs}

hASCs were seeded on silicone rubber BioFlex ${ }^{\circ}$ Culture Plates (Flexcell, USA) at a density of $1.0 \times 10^{5} \mathrm{cells} / \mathrm{ml}$ and incubated in $\alpha$-MEM containing $10 \% \mathrm{FBS}$ at $37^{\circ} \mathrm{C}$ in a $5 \%$ $\mathrm{CO}_{2}$ incubator. Twenty-four hours later, stable adherent cells in the BioFlex ${ }^{\circ}$ Culture Plates were exposed to uniaxial cyclic strain $(5 \%, 0.5 \mathrm{~Hz}, 2 \mathrm{~h} /$ day $)$ by a Flexcell ${ }^{\circ} \mathrm{FX}-$ $5000^{\mathrm{mm}}$ Tension System (Flexcell International Corporation, USA). The control groups were cultured in $\alpha$-MEM supplemented with $10 \% \mathrm{FBS}$ at $37^{\circ} \mathrm{C}$ in a $5 \% \mathrm{CO}_{2}$ incubator for 6 days without cyclic strain loading. On the sixth day, hASCs were collected for real-time quantitative PCR (qPCR) and western blot analyses.

\section{Microarray detection of miRNA changes under cyclic strain}

Total RNA was isolated from hASCs by TRIzol Reagent (Invitrogen, USA) after cyclic strain for 6 days. miRNAs were isolated from total RNA using an Ambion ${ }^{\circ}$ mirVana $^{\text {ix }}$ miRNA Isolation Kit (Thermo Fisher Scientific, USA) according to the manufacturer's instructions. Fluorescent labeling using the T4 RNA ligase labeling method was followed by precipitation with absolute ethanol, and the samples were then blown dry. The isolated RNA was dissolved in hybridization solution and incubated overnight at $42{ }^{\circ} \mathrm{C}$. Then, the samples were washed with $0.2 \%$ sodium dodecyl sulfate (SDS) and $2 \times$ SSC. After drying, the slides were scanned by the LuxScan 10K-A Dual Laser Scanner (BOAO, China).

\section{Bioinformatics analysis}

The conservative characteristics of let-7i-3p, the 3'UTR binding site for LEF1, and the targets of let-7i-3p were analyzed by the following three databases: TargetScan (http://www.targetscan.org), miRWalk (http://mirwalk. umm.uni-heidelberg.de), and miRBase (http://www.mirbase.org).

\section{Artificial regulation of gene expression}

The following genetic research tools were used in this study: LEF1 expression plasmid (EX-LEF1), LEF1 siRNA (siLEF1), negative control expression plasmid (EX-Ctrl), negative control siRNA (siR-Ctrl), let-7i-3p mimic, let-7i-3p inhibitor, negative control of the let-7i-3p mimic (miR-Ctrl mimic) and negative control of the let-7i-3p inhibitor (miR- 
Ctrl inhibitor), LEF1 3'UTR-wild type (wt-LEF1), and LEF1 3'UTR-mutant (mu-LEF1). These vectors were transfected into fourth-passage hASCs using Lipofectamine ${ }^{\mathrm{Tm}} 2000$ Transfection Reagent (Invitrogen, USA) according to the manufacturer's instructions. The let-7i-3p mimic and inhibitor, siLEF1, miR-Ctrl mimic, miR-Ctrl inhibitor, and siR-Ctrl were synthesized by GenePharma Corporation (Shanghai, China). EX-LEF1 (pEZ-M02 vector), EX-Ctrl (pReceiver-M02 vector), wt-LEF1, and mu-LEF1 were supplied by GeneCopoeia Corporation (Guangzhou, China). ORF sequence information for EX-LEF1 and EX-Ctrl is listed in the supplemental materials. Other sequences transfected in this process are listed in Table 1.

To change the expression of $\beta$-catenin, we transduced lentiviral particles containing an shRNA targeting $\beta$ catenin or an shRNA control (Mission Lentiviral Transduction Particles from Sigma-Aldrich, St Louis, MO, USA) into SKOV3 cells. Lentiviral-transduced SKOV3 cells were selected with puromycin $(1.5 \mu \mathrm{M} / \mathrm{mL})$. Transient transfection was performed using a pool of 4 short interfering RNAs (siRNAs) targeting $\beta$-catenin (Dharmacon, Pittsburgh, PA, USA; siGenome SMART pool) or individual siRNA sequences (\#1: GCGUUUGGCU GAACCAUCA and \#2: UAAUGAGGACCUAUACUUA, Dharmacon) and DreamFECT transfection reagent $(\mathrm{Oz}$ Biosciences, Marseille, France). The scrambled siRNA pool (Dharmacon) was used as a control.

\section{Dual-luciferase reporter assay}

Sequence fragments, such as LEF1 3'UTR-WT and LEF1 3'UTR-Mu, were inserted between the NotI and XhoI cleavage sites of the psiCHECK-2 vector (Promega, Madison, WI, USA) downstream of the Renilla luciferase reporter gene. The sequences mentioned above are listed in Additional file 1: Table S1. HEK-293 T cells were seeded into 96 -well plates at $70 \%$ confluence and then cotransfected with each reporter construct (pmirGLOLEF1-WT and pmirGLO LEF1-Mu) and Lv-let-7i-3p, Lv-miR-NC, Lv-ASO-let-7i-3p, or LvASO-NC. The Dual-Luciferase Reporter Gene Assay Kit (Beyotime, Shanghai, China) was used to detect the firefly and
Renilla luciferase activities $48 \mathrm{~h}$ after transfection. The firefly values were normalized to Renilla luciferase.

\section{Effect of let-7i-3p on LEF1 in hASCs transfected with the let-7i-3p mimic and inhibitor as determined by immunofluorescence}

The fourth-passage hASCs transfected with the let-7i-3p mimic and inhibitor were used to detect the effect of let7i-3p on LEF1 by immunofluorescence. Two days after transfection, hASCs were seeded on coverslips in 9-well plates. After $48 \mathrm{~h}$, hASCs were fixed with $4 \%$ paraformaldehyde for $15 \mathrm{~min}$ and washed three times with PBS. Triton X-100 (0.5\%, Sigma-Aldrich, USA) was added to each well for $20 \mathrm{~min}$ at room temperature. After rinsing three times with PBS, hASCs were blocked with goat serum for $2 \mathrm{~h}$. Primary antibodies specific for LEF1 (1:1000, R\&D Systems, USA) were added to the cells and then incubated overnight at $4{ }^{\circ} \mathrm{C}$. Fluorescent $\mathrm{Cy} 3$ secondary antibodies (1:50, Proteintech, USA) were added and incubated for $1 \mathrm{~h}$ at $37^{\circ} \mathrm{C}$ in the dark after rinsing three times with PBS Tween-20. The nucleus was then restained with 4',6-diamidino-2-phenylindole (DAPI, Sigma-Aldrich, USA). Cells were subsequently viewed by fluorescence microscopy (ZEISS, Oberkochen, Germany).

\section{Gene expression detection by qPCR}

Total RNA was isolated from hASCs by TRIzol Reagent and detected by a NanoVue ${ }^{\mathrm{TN}}$ Plus spectrophotometer (GE Healthcare Life Sciences, USA) to determine purity at $260 / 280 \mathrm{~nm}$. cDNA was synthesized from RNA using PrimeScript RT Master Mix (Perfect Real-Time, TaKaRa, Japan). PCR was performed on the ABI 7300 Real-Time PCR System (Applied Biosystems, UK). The following markers associated with osteogenesis and the Wnt/B-catenin pathway alkaline phosphatase (ALP), runt-related transcription factor 2 (RUNX2), secreted protein acidic and cysteine-rich (SPARC), LEF1, $\beta$-catenin, and glyceraldehyde-3-phosphate dehydrogenase (GAPDH). All primer sequences used in this study are shown in Table 2. The results of qPCR are shown as $\mathrm{Ct}$ values and compared with GAPDH.

Table 1 The constructed sequences used in this study

\begin{tabular}{|c|c|c|}
\hline Genes & Sequence & \\
\hline let-7i-3p-503-3p mimic & & 5'-CUGCGCAAGCUACUGCCUUGCUdTdT-3' \\
\hline miR-Ctrl mimic & & 5'-UUGUACUACACAAAAGUACUGdTdT-3' \\
\hline let-7i-3p inhibitor & & 5'-AGCAAGGCAGUAGCUUGCGCAGdTdT-3' \\
\hline miR-Ctrl inhibitor & & 5'-CAGUACUUUUGUGUAGUACAAdTdT-3' \\
\hline \multirow[t]{2}{*}{ SiLEF1 } & Sense & 5'-CCGUGAAGAGCAGGCUAAATTdTdT-3' \\
\hline & Antisense & 5'-UUUAGCCUGCUCUUCACGGTTdTdT-3' \\
\hline \multirow[t]{2}{*}{ siR-Ctrl } & Sense & 5'-UUCUCCGAACGUGUCACGUdTdT-3' \\
\hline & Antisense & 5'-ACGUGACACGUUCGGAGAAdTdT-3' \\
\hline
\end{tabular}


Table 2 The primer sequences used for qPCR in this study

\begin{tabular}{|c|c|c|c|}
\hline Gene & Accession no. & $5^{\prime}-3^{\prime}$ & $\operatorname{Tm}\left({ }^{\circ} \mathrm{C}\right)$ \\
\hline \multirow[t]{2}{*}{ RUNX2 } & NM_001015051 & F: TAGATAGTGATTGCGTTTGGCTATG & 60 \\
\hline & & R: CACTAAGAAATGTTCAAGGGTCC & 60 \\
\hline \multirow[t]{2}{*}{ ALP } & NM_003064 & F: GAAAGTCCTTCAAAGCTGGAGTCT & 60 \\
\hline & & R: TCTGGCACTCAGGTTTCTTGTATC & 60 \\
\hline \multirow[t]{2}{*}{ SPARC } & NM_001309443 & F: TGTGATCTAAATCCACTCCTTCCA & 60 \\
\hline & & R: ACAAACCATCCAAACATTITAAACA & 60 \\
\hline \multirow[t]{2}{*}{ LEF1 } & NM_001130714 & F: TGCCAAATATGAATAACGACCCA & 60 \\
\hline & & R: GAGAAAAGTGCTCGTCACTGT & 60 \\
\hline \multirow[t]{2}{*}{$\beta$-catenin } & NM_001098209 & F: AAAATGGCAGTGCGTTAAG & 60 \\
\hline & & R: TTTGAAGGCAGTCTGTCGTA & 60 \\
\hline \multirow[t]{2}{*}{ GAPDH } & NM_001256799.2 & F: GAACGGGAAGCTCACTGG & 60 \\
\hline & & R: GCCTGCTTCACCACCTTCT & 60 \\
\hline
\end{tabular}

\section{Protein expression detection by western blot}

Adherent hASCs were lysed with cell lysis buffer for western blot and immunoprecipitation (IP) analyses (Beyotime, China) and Nuclear and Cytoplasmic Protein Extraction Kit (Beyotime, China) respectively. Then, samples centrifuged at $4{ }^{\circ} \mathrm{C}(16,000 g, 20 \mathrm{~min})$. The protein concentrations were determined using a BCA Protein Assay Kit (Beyotime, China). The protein was boiled at $100{ }^{\circ} \mathrm{C}$ for $5 \mathrm{~min}$ after the addition of $5 \times$ SDS loading buffer (Beyotime, China). Protein samples were loaded onto SDS polyacrylamide gel electrophoresis gels and then electrophoresed at $100 \mathrm{~V}$ for $1.5 \mathrm{~h}$. Then, the protein blots were transferred onto membranes (GE Healthcare Life Sciences, USA), which were blocked with TBS containing $5 \%$ nonfat milk overnight at $4{ }^{\circ} \mathrm{C}$. Then, the membranes were incubated with primary antibodies specific for ALP (1:1000, Abcam, USA), RUNX2 (1:1000, Abcam, USA), SPARC (1:1000, Abcam, USA), LEF1 (1: 1000, R\&D Systems, USA), $\beta$-catenin (1:1000, Abcam, USA), and GAPDH (1:1000, Cell Signaling Technology, USA) overnight at $4{ }^{\circ} \mathrm{C}$. Afterward, the membranes were washed three times with TBS-0.05\% Tween 20 and incubated with the corresponding secondary antibodies for 1 $\mathrm{h}$ at room temperature. Next, ECL solution (Thermo Fisher Scientific, Germany) was added to the membranes and incubated for $1 \mathrm{~min}$. The protein blots were visualized by exposure to enhanced chemiluminescence reagents (GE Healthcare, USA). Grayscales on the blots were analyzed by Quantity One software (Bio-Rad, Hercules, CA, USA).

\section{Statistical analysis}

Statistical values were calculated using SPSS 20.0 software (IBM SPSS Statistics, Armonk, NY: IBM-Corp.) One-way analysis of variance (ANOVA) was used for multiple group comparisons. Two-way ANOVA was used for two-group comparisons. All of the results are presented as the mean \pm sem, and statistical significance was set at $p<0.05$.

\section{Results}

Cell growth and cycle of hASCs

As shown by a light microscope (magnification $\times 100$; Leica Microsystems $\mathrm{GmbH}$, Germany), the fourthpassage hASCs had a typical fibroblast-like morphology (Fig. 1a). The growth curve was drawn based on the results of CCK- 8 assays (Fig. 1b). In the first 3 days, hASCs were in the slow growth phase; from the third to sixth days, they entered the exponential growth phase; and at the seventh day, they were in the plateau phrase. The cell cycle of hASCs was detected by flow cytometry, revealing that $82.12 \% \pm 5.83 \%$ of the cells were in G1 phase, and $17.88 \% \pm 2.59 \%$ of the cells were in S/G2 phase (Fig. 1c).

\section{Immunophenotypes and differentiation of hASCs}

Flow cytometry was used to determine the immunophenotypes of hASCs. The cells were positive for CD29 $(99.81 \% \pm 0.76 \%), \quad$ CD90 $(98.43 \% \pm 1.77 \%)$, and CD105 $(92.21 \% \pm 6.48 \%)$ and negative for CD34 (2.39\% $\pm 0.72 \%)$ and CD45 (0.97\% $\pm 0.41 \%)$ (Fig. 1d).

The mineralized nodules in hASCs, which were cultured in the osteogenic medium after 21 days, were also stained by Alizarin Red (Fig. 1e). The lipid droplets in hASCs, which were cultured in the adipogenic medium after 14 days, were stained by Oil Red O (Fig. 1e). The proteoglycans in hASCs, which were cultured in the adipogenic medium after 14 days, were stained by Alcian Blue to confirm the existence of proteoglycans (Fig. 1e).

\section{The osteogenic differentiation of hASCs under cyclic strain}

The osteogenic differentiation and Wnt/ $\beta$-catenin pathway of hASCs, which were under cyclic strain for 6 days, 


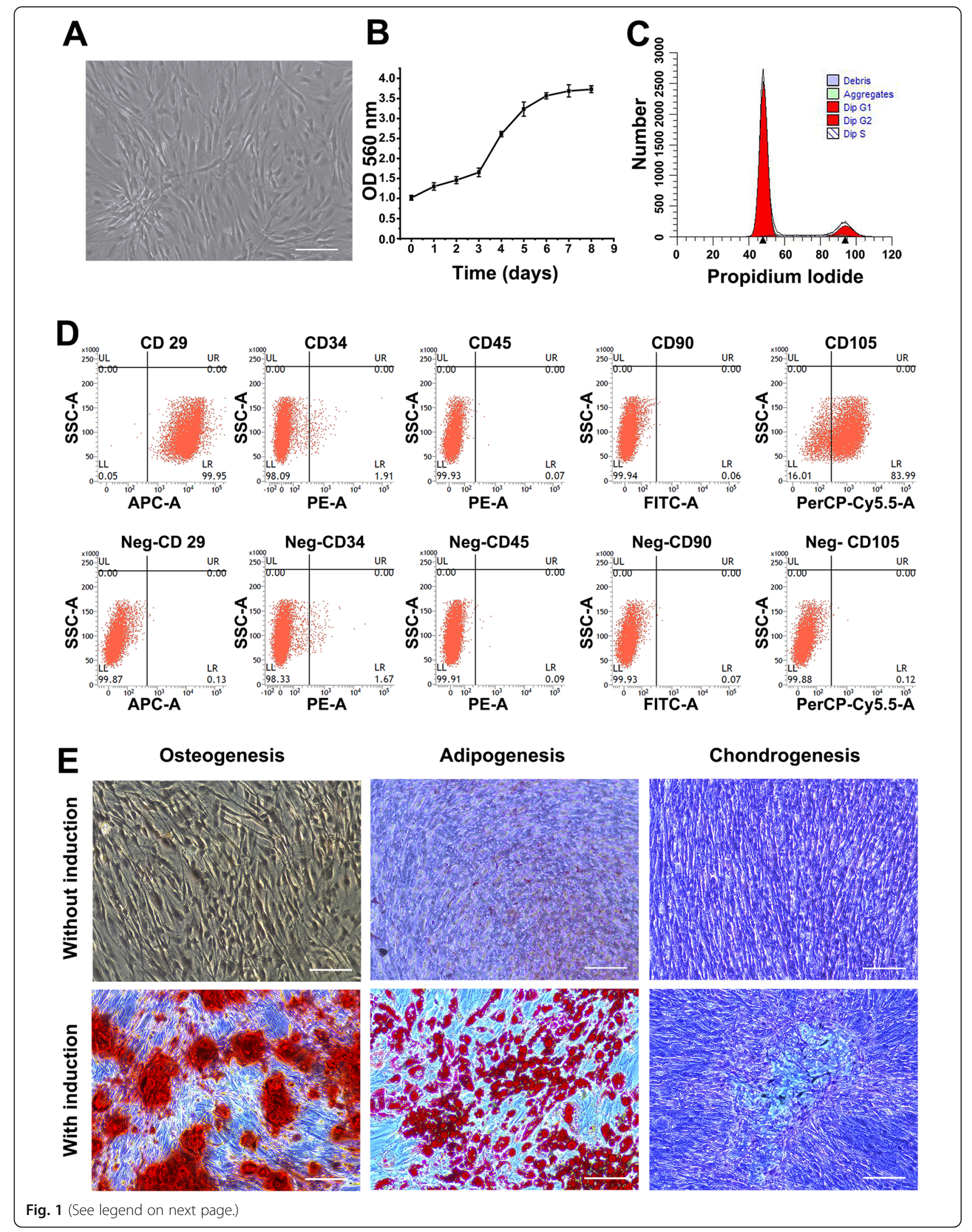


(See figure on previous page.)

Fig. 1 The characteristics of hASCs. a The fourth-passage hASCs screened by light microscopy (scale bar $100 \mu \mathrm{m}$ ). b The growth curve was determined by CCK-8 assays. hASCs were in the slow growth phase from the first to the third days, exponential growth phase from the third to the sixth days, and the plateau phrase from the seventh d. c hASC cell cycle analysis by flow cytometry: G1 phase (82.12\% $\pm 5.83 \%)$ and S/G2 phase (17.88\% $\pm 2.59 \%)$. $\mathbf{d}$ Immunophenotypes of hASCs detected by flow cytometry: CD29(+) (99.81\% $\pm 0.76 \%)$, CD90(+) $(98.43 \% \pm 1.77 \%)$, CD105(+) $(92.21 \% \pm 6.48 \%)$; CD34(-) $(2.39 \% \pm 0.72 \%), C D 45(-)(0.97 \% \pm 0.41 \%)$. e Multilineage of hASCs induced by media. The mineralized nodules were stained with Alizarin Red. The lipid droplets were stained with Oil Red O. The proteoglycans were stained with Alcian Blue (scale bar $100 \mu \mathrm{m}$ )

were analyzed by qPCR and western blot. All the following results were compared to those obtained using hASCs cultured in $\alpha$-MEM with $10 \%$ FBS at $37^{\circ} \mathrm{C}$ and $5 \% \mathrm{CO}_{2}$ without cyclic strain.

By qPCR analysis, RUNX2, ALP, and SPARC, which are associated with osteogenic differentiation, were significantly increased $2.47 \pm 0.73$-fold $(p=0.011), 2.57 \pm$ 0.09 -fold $(p=0.004)$, and $2.13 \pm 0.60$-fold $(p=0.018)$, respectively; LEF1 and $\beta$-catenin, which are associated with the $\mathrm{Wnt} / \beta$-catenin pathway, were increased $1.89 \pm$ 0.45 -fold $(p=0.012)$ and $2.22 \pm 0.51$-fold $(p=0.017)$, respectively (Fig. 2a).

Western blot analysis showed that RUNX2, ALP, and SPARC levels were significantly increased $2.59 \pm 0.31$ fold $(p=0.001), 1.39 \pm 0.09$-fold $(p=0.010)$, and $1.79 \pm$ 0.21 -fold $(p=0.005)$, respectively; LEF1 and $\beta$-catenin levels were increased $1.55 \pm 0.39$-fold $(p=0.030)$ and $1.63 \pm 0.20$-fold $(p=0.004)$, respectively (Fig. $2 \mathrm{~b}$ ).

\section{Microarray detection of miRNA changes under cyclic strain}

After the exertion of cyclic strain on hASCs for 6 days, the microarray analysis of miRNAs showed that the expression levels of 237 miRNAs in hASCs were significantly changed ( $>$ 2-fold). Among them, 150 miRNAs exhibited decreased in expression, and 87 miRNAs exhibited increased expression. Some miRNAs associated with $\mathrm{Wnt} / \beta$-catenin and their fold changes are shown in Fig. 3. The complete microarray results depicting miRNA changes are shown in Additional file 2: Figure S1.

\section{LEF1 is a target of let-7i-3p}

The predicted binding sequences of both the 3'UTR in LEF1 and let-7i-3p are highly conserved across species (Fig. 4a, b). The LEF1 3'UTR, which is a component of the Wnt/ $\beta$-catenin signaling pathway, was likely matched to let-7i-3p (Fig. 4a).

Overexpression of let-7i-3p significantly inhibited the luciferase activity of pMIR/LEF1-WT reporter genes, and the luciferase activity was decreased by $2.19 \pm 0.49$ fold ( $p=0.007$ ) compared with that in the control group, which was a significant difference. In the reporter gene system in which the LEF1 mRNA 3'UTR binding sites were mutated, overexpression of let-7i-3p did not affect the reporter gene luciferase activity. Inhibition of let-7i$3 p$ expression induced the luciferase activity of the
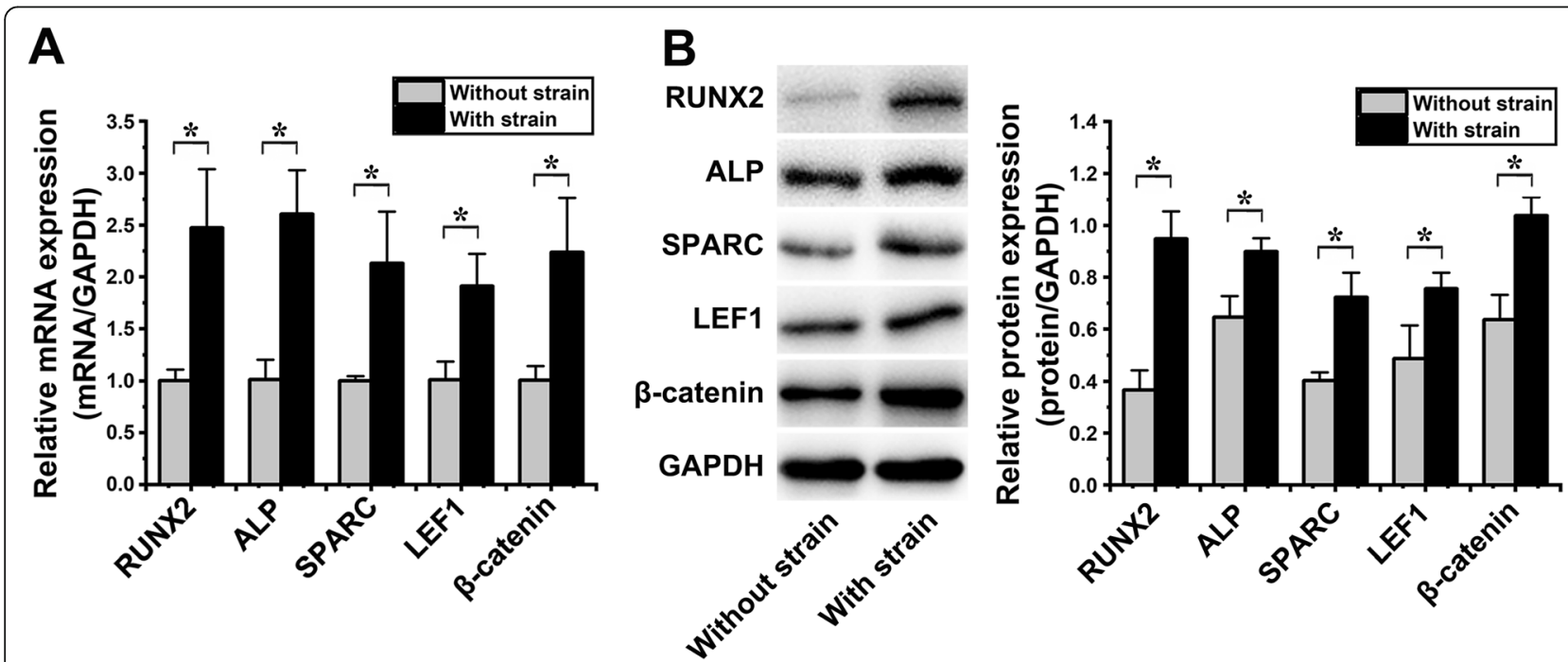

Fig. 2 The osteogenic differentiation of hASCs under cyclic strain $\left({ }^{*} p<0.05\right.$, significant differences existed between these two groups). a $q P C R$ results. The mRNA levels of RUNX (22.47 \pm 0.73 -fold, $p=0.011)$, ALP (2.57 \pm 0.09 -fold, $p=0.004)$, SPARC (2.13 \pm 0.60 -fold, $p=0.018)$, LEF1 (1.89 \pm 0.45 -fold, $p=0.012)$, and $\beta$-catenin ( $2.22 \pm 0.51$-fold, $p=0.017)$ were significantly increased. $\mathbf{b}$ Western blot results. The pictures of protein blots are shown in the left column. The protein expression levels of RUNX2 (2.59 \pm 0.31 -fold, $p=0.001)$, ALP (1.39 \pm 0.09 -fold, $p=0.010)$, SPARC (1.79 \pm 0.21 fold, $p=0.005)$, LEF1 $(1.55 \pm 0.39$-fold, $p=0.030)$, and $\beta$-catenin $(1.63 \pm 0.20$-fold, $p=0.004)$ were significantly increased 


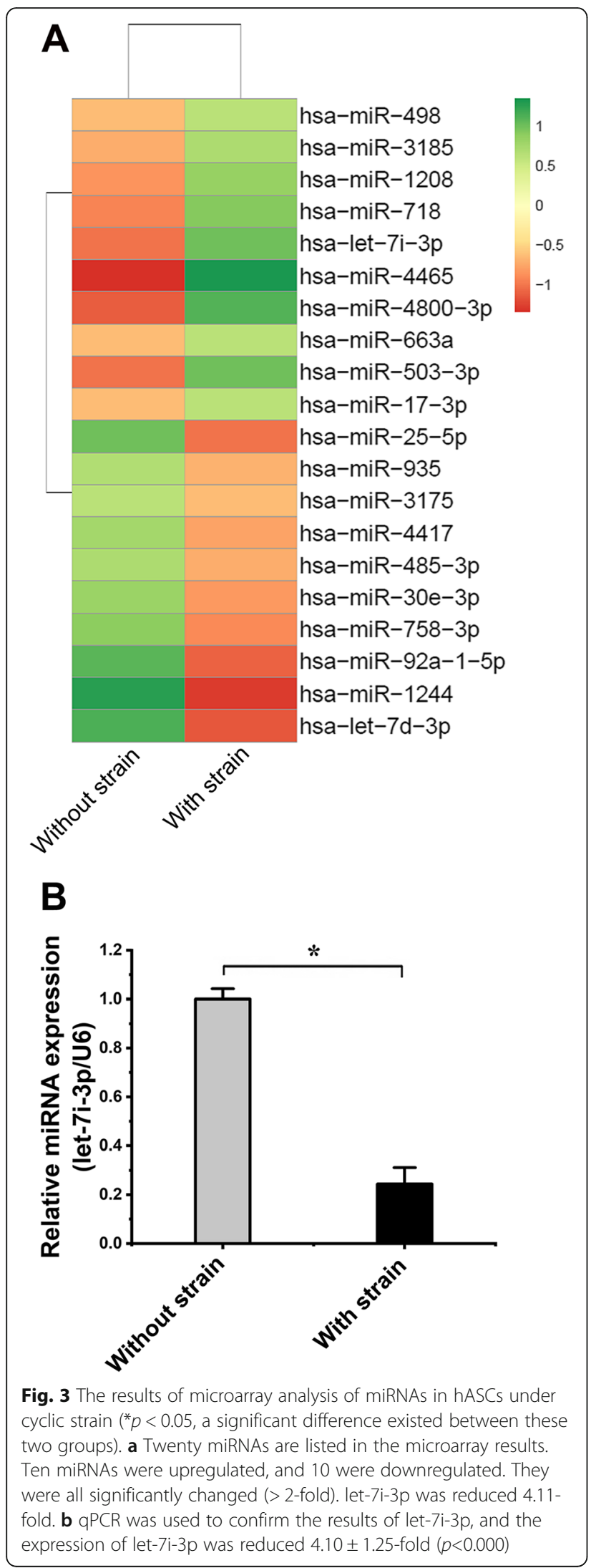

reporter gene. Compared with that in the control group, the luciferase activity in the let-7i-3p inhibition group was increased $1.58 \pm 0.37$-fold ( $p=0.011$ ), and the difference was significant. Then, combination with the LEF1 mRNA 3'UTR was assessed. In the reporter gene system with a site mutation, inhibition of let-7i-3p expression did not affect the reporter gene luciferase activity. The results are shown in Fig. $4 \mathrm{c}$ and d.

LEF1 mRNA expression in hASCs transfected with let$7 \mathrm{i}$-3p mimic was decreased $2.43 \pm 0.24$-fold $(p=0.013)$, as shown by qPCR, compared to that in hASCs transfected with the miR-Ctrl mimic. LEF1 mRNA expression in hASCs transfected with the let-7i-3p inhibitor was increased $1.66 \pm 0.07$-fold ( $p=0.002$ ), as shown by qPCR, compared to that in hASCs transfected with the miRCtrl inhibitor (Fig. 4e). LEF1 protein expression in hASCs transfected with let-7i-3p mimic was decreased $1.92 \pm 0.63$-fold $(p=0.016)$, as shown by western blot, compared to that in hASCs transfected with the miRCtrl mimic. LEF1 protein expression in hASCs transfected with the let-7i-3p inhibitor was increased $1.43 \pm$ 0.10 -fold $(p=0.013)$, as shown by western blot, compared to that in hASCs transfected with the miR-Ctrl inhibitor (Fig. 4f). Together, these results suggest that LEF1 is a target gene of let-7i-3p.

\section{Immunofluorescence analysis of hASCs transfected with let-7i-3p mimics and inhibitor}

The immunofluorescence intensity of LEF1 and $\beta$ catenin in hASCs was inhibited after transfection of let$7 \mathrm{i}-3 \mathrm{p}$ mimics compared with that in normal cultured hASCs (Fig. 5). The immunofluorescence intensity of LEF1 and $\beta$-catenin in hASCs was promoted after transfection of the let-7i-3p inhibitor compared with that in normal cultured hASCs (Fig. 5a). The immunofluorescence intensity of $\beta$-catenin in both the cytoplasm and nucleus of hASCs was promoted after transfection of the let-7i-3p inhibitor compared with that in normal cultured hASCs (Fig. 5b).

\section{Effect of LEF1 on the osteogenic differentiation of hASCs under cyclic strain}

To determine the transfection efficiency, the expression of LEF1 was detected by qPCR after transfection of EXLEF1, siLEF1, EX-Ctrl, and siR-Ctrl into hASCs. Compared to that in the EX-Ctrl group, the LEF1 expression in the EX-LEF1 group was increased $3.37 \pm 1.24$-fold $(p=0.009)$ (Fig. 6a). Compared to that in the siR-Ctrl group, the LEF1 expression in the siLEF1 group was significantly decreased $2.56 \pm 0.19$-fold $(p<0.001)$ (Fig. 6a).

qPCR analysis revealed the effect of LEF1 on the osteogenic differentiation of hASCs under cyclic strain. Compared to those in the EX-Ctrl group, the RUNX2, ALP, SPARC, LEF1, and $\beta$-catenin levels in hASCs 


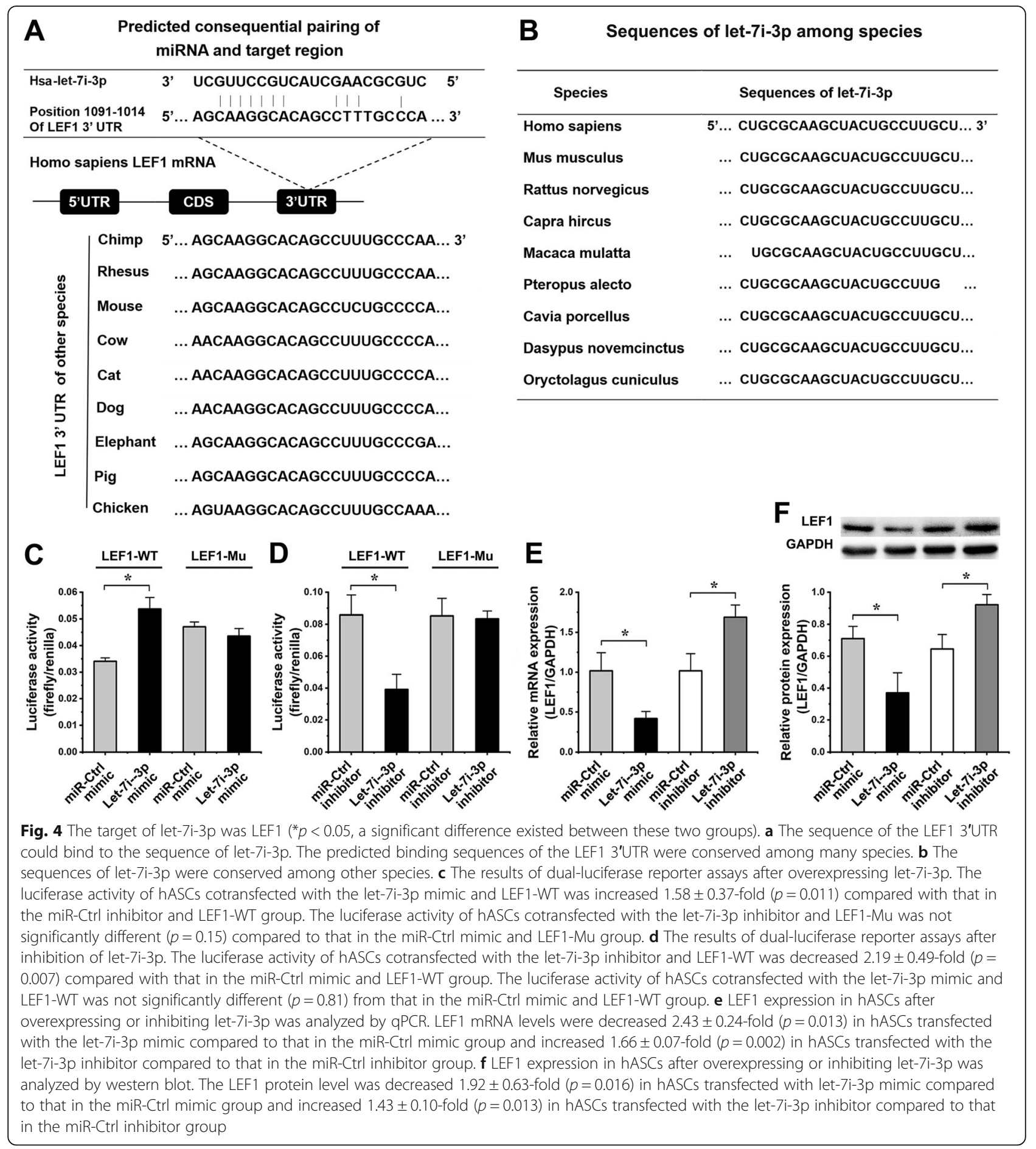

transfected with EX-LEF1 and then exposed to loading cyclic strain for 6 days were significantly increased $4.54 \pm 2.64$-fold $(p<0.001), 3.38 \pm 0.64$-fold $(p=0.002)$, $2.07 \pm 0.35$-fold $(p=0.008), 4.67 \pm 0.47$-fold $(p=0.008)$, and $2.33 \pm 0.39$-fold $(p=0.023)$, respectively (Fig. 6b). Compared to those in the siR-Ctrl group, the RUNX2, ALP, SPARC, LEF1 and $\beta$-catenin levels in hASCs transfected with siLEF1 and then exposed to loading cyclic strain for 6 days were decreased $2.11 \pm 0.33$-fold $(p<0.001), 1.65 \pm 0.68$-fold $(p=0.044), 3.04 \pm 1.38$-fold $(p=0.039), \quad 3.40 \pm 1.18$-fold $(p=0.001), \quad$ and $2.72 \pm$ 0.44 -fold $(p=0.003)$, respectively (Fig. $6 \mathrm{c}$ ).

As shown by the above results, the osteogenic differentiation of hASCs was induced by overexpression of LEF1. In contrast, the osteogenic differentiation of hASCs was inhibited by knockdown of LEF1. 


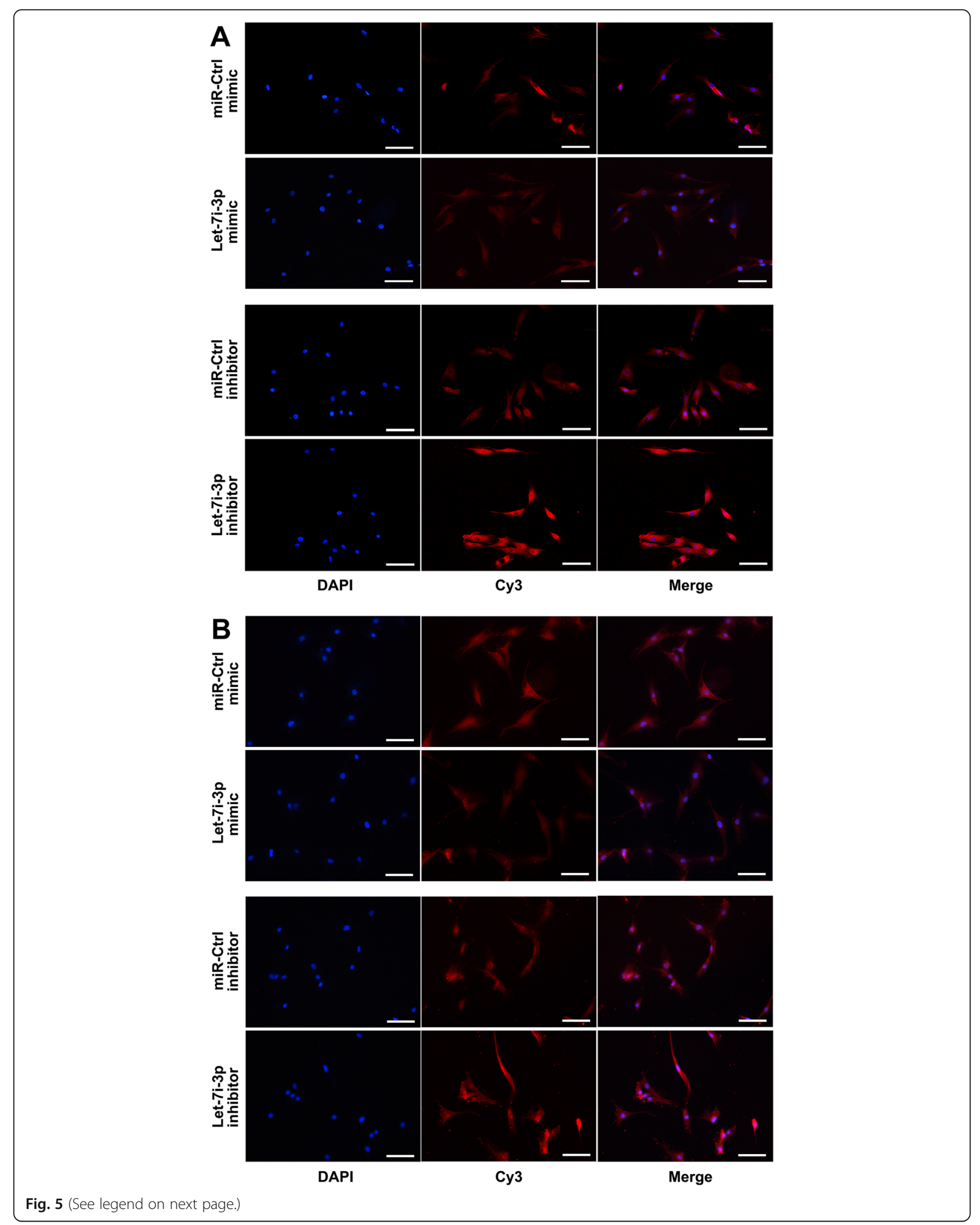


(See figure on previous page.)

Fig. 5 Immunofluorescence analysis of hASCs transfected with let-7i-3p mimics and inhibitor. a Compared to that in normal cultured hASCs, the immunofluorescence intensity of LEF1 was decreased in hASCs transfected with let-7i-3p mimics. Compared with that in normal cultured hASCs, the immunofluorescence intensity of LEF1 was increased in hASCs transfected with the let-7i-3p inhibitor. $\mathbf{b}$ Compared to that in normal cultured hASCs, the immunofluorescence intensity of $\beta$-catenin was decreased in both the cytoplasm and nucleus of hASCs transfected with let-7i-3p mimics. Compared to that in normal cultured hASCs, the immunofluorescence intensity of $\beta$-catenin in both the cytoplasm and nucleus was promoted after transfection of the let-7i-3p inhibitor (scale bar $100 \mu \mathrm{m}$ )

\section{Effect of $\beta$-catenin on the osteogenic differentiation of} hASCs under cyclic strain

To determine the transfection efficiency, the expression of $\beta$ catenin was detected by qPCR after transfection of EX- $\beta$ catenin, si $\beta$-catenin, EX-Ctrl, and siR-Ctrl into hASCs. Compared to that in the EX-Ctrl group, the $\beta$-catenin expression in the EX- $\beta$-catenin group was increased $3.71 \pm 0.42$-fold $(p<0.001)$ (Fig. 7a). Compared to that in the siR-Ctrl group, the $\beta$-catenin expression in the si $\beta$-catenin group was significantly decreased $3.00 \pm 0.76$-fold $(p=0.002)$ (Fig. 7a).

qPCR analysis revealed the effect of $\beta$-catenin on the osteogenic differentiation of hASCs under cyclic strain. Compared to those in the EX-Ctrl group, the levels of RUNX2, ALP, SPARC, LEF1, and $\beta$-catenin in hASCs transfected with EX- $\beta$-catenin and then exposed to loading cyclic strain for 6 days were significantly increased $4.73 \pm 1.60$-fold $(p=0.001), 3.76 \pm 2.04$-fold $(p=0.021)$, $1.85 \pm 0.49$-fold $(p=0.004), 2.64 \pm 0.40$-fold $(p=0.006)$, and $4.30 \pm 1.12$-fold $(p=0.001)$, respectively (Fig. 7b). Compared to those in the siR-Ctrl group, the levels of
RUNX2, ALP, SPARC, LEF1, and $\beta$-catenin in hASCs transfected with si $\beta$-catenin and then exposed to loading cyclic strain for 6 days were decreased $2.61 \pm 0.45$-fold $(p<0.001), 3.46 \pm 0.79$-fold $(p=0.009), 2.16 \pm 0.02$-fold $(p=0.010), 2.52 \pm 0.61$-fold $(p=0.001)$, and $4.15 \pm 1.72$ fold $(p=0.004)$, respectively (Fig. 7c).

As shown by the above results, the osteogenic differentiation of hASCs was induced by overexpression of $\beta$-catenin. In contrast, the osteogenic differentiation of hASCs was inhibited by knockdown of $\beta$-catenin.

\section{Effects of let-7i-3p on the osteogenic differentiation of hASCs under cyclic strain}

To determine the transfection efficiency, the expression of let-7i-3p in hASCs was detected by qPCR after transfection with the let-7i-3p mimic and inhibitor, miR-Ctrl mimic, and miR-Ctrl inhibitor. Compared to that in the miR-Ctrl mimic group, the expression of let-7i-3p in the let-7i-3p mimic group was increased $776.40 \pm 111.78$-fold $(p<0.001)$ (Fig. 8a). Compared to that in the miR-Ctrl inhibitor group,

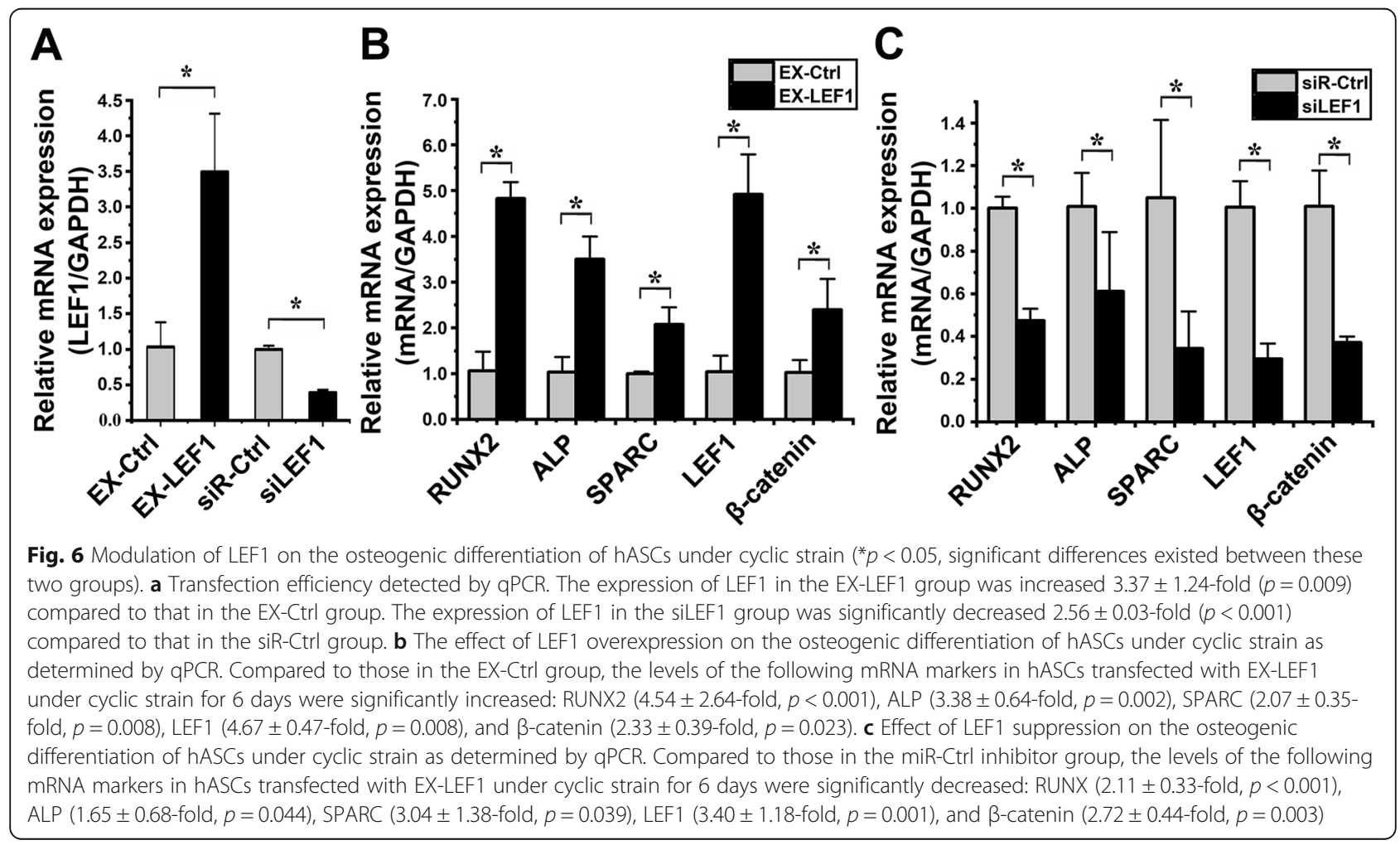



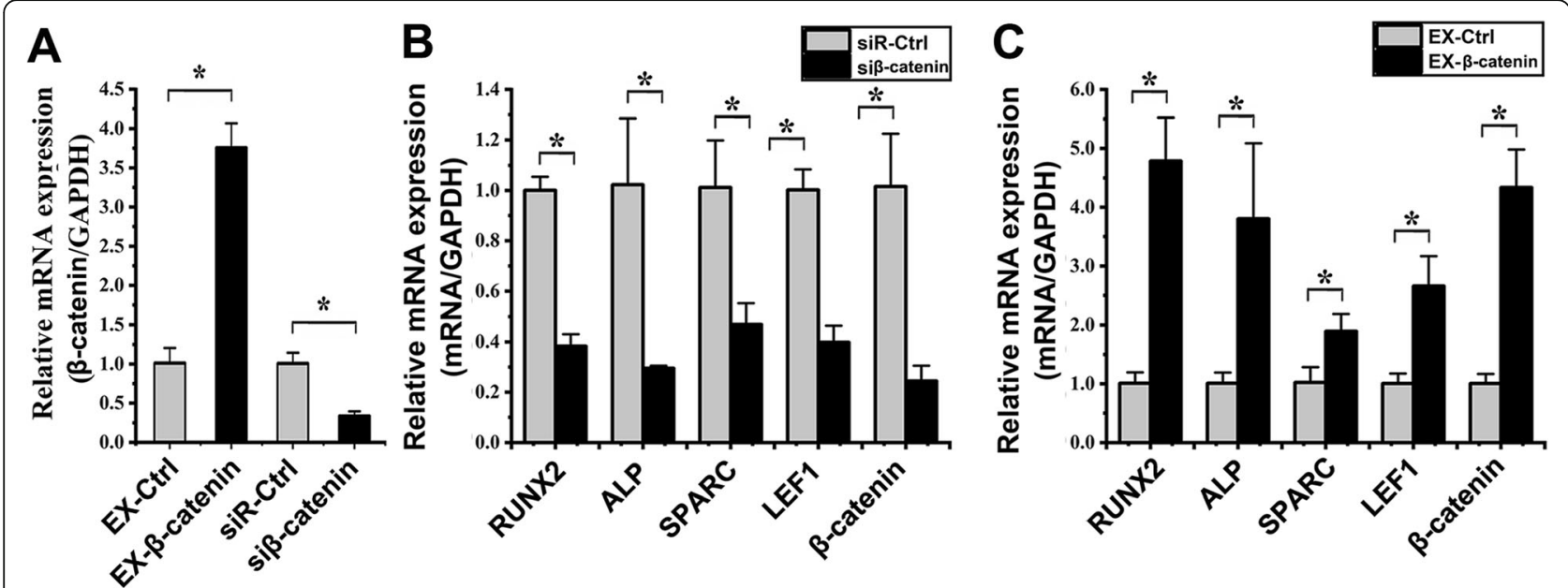

Fig. 7 Effects of $\beta$-catenin modulation on the osteogenic differentiation of hASCs under cyclic strain $\left({ }^{*} p<0.05\right.$, significant differences existed between these two groups). a Transfection efficiency detected by qPCR. The expression of $\beta$-catenin in the EX- $\beta$-catenin group was increased $3.71 \pm 0.42$-fold $(p<0.001)$ compared to that in the EX-Ctrl group. The expression of $\beta$-catenin in the si $\beta$-catenin group was significantly decreased $3.00 \pm 0.76$-fold $(p=0.002$ ) compared to that in the siR-Ctrl group. $\mathbf{b}$ The effect of $\beta$-catenin overexpression on the osteogenic differentiation of hASCs under cyclic strain as determined by qPCR. Compared to those in the EX-Ctrl group, the levels of the following mRNA markers in hASCs transfected with EX- $\beta$-catenin under cyclic strain for 6 days were significantly increased: RUNX2 (4.73 $\pm 1.60-$ fold, $p=0.001)$, ALP (3.76 \pm 2.04 -fold, $p=0.021)$, SPARC (1.85 \pm 0.49 -fold, $p=0.004$ ), LEF1 ( $2.64 \pm 0.40$-fold, $p=0.006)$, and $\beta$-catenin $(4.30 \pm 1.12$-fold, $p=0.001)$. c The effect of $\beta$-catenin suppression on the osteogenic differentiation of hASCs under cyclic strain as determined by qPCR. Compared to those in the miR-Ctrl inhibitor group, the levels of the following mRNA markers in hASCs transfected with EX- $\beta$-catenin under cyclic strain for 6 days were significantly decreased: RUNX (2.61 \pm 0.45 -fold, $p<0.001)$, ALP (3.46 \pm 0.79 -fold, $p=0.009)$, SPARC (2.16 \pm 0.02 -fold, $p=0.010)$, LEF1 (2.52 \pm 0.61 -fold, $p=0.001)$, and $\beta$-catenin $(4.15 \pm 1.72$-fold, $p=0.004)$

the expression of let-7i-3p in the let-7i-3p inhibitor group was decreased $3.88 \pm 1.36$-fold ( $p=0.005$ ) (Fig. 8b).

qPCR analysis revealed the effect of let-7i-3p on the osteogenic differentiation of hASCs under cyclic strain. Compared to those in the miR-Ctrl mimic group, the levels of RUNX2, ALP, SPARC, LEF1, and $\beta$-catenin in hASCs transfected with the let-7i-3p mimic and then exposed to loading cyclic strain for 6 days were significantly decreased $3.52 \pm 1.94$-fold $(p=0.002), \quad 1.80 \pm 0.15$-fold $(p=0.012), \quad 3.07 \pm 0.86$-fold $(p<0.001), \quad 2.08 \pm 0.73$-fold $(p=0.018)$, and $3.60 \pm 0.57$-fold $(p=0.002)$, respectively (Fig. 8c). The immunofluorescence intensity of $\beta$-catenin in the hASC nucleus was also decreased in hASCs transfected with the let-7i-3p mimic (Fig. 8d). Compared to those in the miR-Ctrl mimic group, the levels of cytoplasmic and nuclear $\beta$-catenin in hASCs transfected with the let-7i-3p mimic significantly decreased $1.56 \pm 0.06$-fold $(p=0.002)$ and $1.74 \pm 0.24$-fold $(p=0.019)$, respectively (Fig. 8e).

Compared to those in the miR-Ctrl inhibitor group, the levels of RUNX2, ALP, SPARC, LEF1, and $\beta$-catenin in hASCs transfected with the let-7i-3p inhibitor and then exposed to loading cyclic strain for 6 days were increased $3.58 \pm 0.82$-fold $(p=0.009), 2.53 \pm 0.49$-fold ( $p=$ $0.008), 1.83 \pm 0.28$-fold $(p=0.012), 2.31 \pm 1.03$-fold ( $p=$ $0.046)$, and $2.86 \pm 1.68$-fold $(p=0.010)$, respectively (Fig. 8f). The immunofluorescence intensity of $\beta$-catenin in the hASC nucleus was also increased in hASCs transfected with the let-7i-3p inhibitor (Fig. 8g). Compared to those in the miR-Ctrl inhibitor group, the levels of cytoplasmic and nuclear $\beta$-catenin in hASCs transfected with the let-7i-3p inhibitor significantly increased $1.45 \pm 0.11$-fold $(p=0.003)$ and $2.97 \pm 0.19$-fold $(p=0.001)$, respectively (Fig. 8h).

As shown by the above results, the osteogenic differentiation of hASCs was promoted by the suppression of let-7i-3p. In contrast, the osteogenic differentiation of hASCs was inhibited by overexpression of let-7i-3p.

\section{Effect of LEF1 regulated by let-7i-3p on the osteogenic differentiation of hASCs under cyclic strain}

Compared to those in the group transfected with the let7i-3p mimic and EX-Ctrl, the mRNA expression levels of RUNX2, ALP, SPARC, LEF1, and $\beta$-catenin were increased $2.10 \pm 0.69$-fold $(p=0.014), 2.80 \pm 1.62$-fold $(p=$ $0.005), 2.06 \pm 0.30$-fold $(p=0.006), 3.06 \pm 0.52$-fold $(p=$ $0.029)$, and $2.75 \pm 0.68$-fold $(p=0.010)$, respectively, in hASCs cotransfected with the let-7i-3p mimic and EXLEF1 (Fig. 9a); the protein expression levels of the above markers were also increased $2.02 \pm 0.38$-fold $(p=0.004)$, $2.03 \pm 0.07$-fold $(p=0.002), 2.54 \pm 0.60$-fold $(p=0.004)$, $2.91 \pm 0.61$-fold $(p=0.004)$, and $1.49 \pm 0.17$-fold $(p=$ 0.022), respectively (Fig. 9b). The immunofluorescence intensity of $\beta$-catenin in the hASC nucleus was also increased in hASCs cotransfected with the let-7i-3p mimic and EX-LEF1 (Fig. 9c). Compared to those in the group 


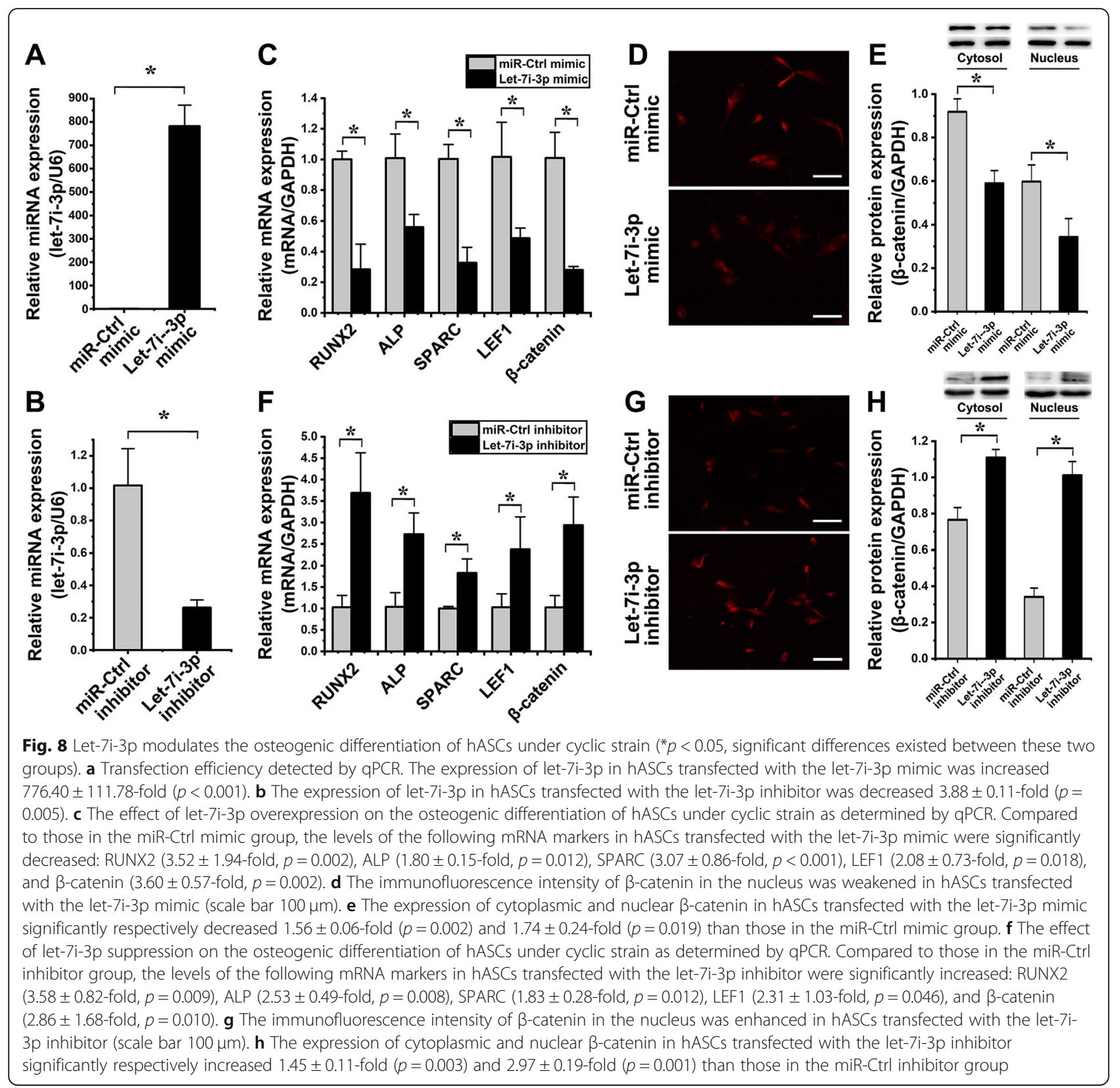

transfected with the let-7i-3p mimic and EX-Ctrl, the protein expression levels of cytoplasmic and nuclear $\beta$ catenin were increased $1.30 \pm 0.10$-fold $(p=0.026)$ and $1.40 \pm 0.15$-fold $(p=0.017)$, respectively, in hASCs cotransfected with the let-7i-3p mimic and EX-LEF1 (Fig. 9d). Therefore, the inhibitory effect of the let-7i-3p mimic on the osteogenic differentiation of hASCs could be relieved by LEF1 overexpression under cyclic strain.

Compared to those in the group transfected with the let-7i-3p inhibitor and siR-Ctrl, the mRNA levels of the above markers were decreased 1.52 \pm 0.26 -fold ( $p=$ 0.026 ), $3.12 \pm 1.55$-fold $(p=0.002), 2.55 \pm 0.25$-fold ( $p=$ $0.038), 3.92 \pm 0.72$-fold $(p=0.018)$, and $2.25 \pm 1.24$-fold $(p=0.030)$, respectively, in hASCs cotransfected with the let-7i-3p inhibitor and siLEF1 (Fig. 9e); the protein expression levels of the above markers were also decreased $2.00 \pm 0.42$-fold $(p=0.002), 1.54 \pm 0.17$-fold $(p=0.013)$, $1.71 \pm 0.19$-fold $(p=0.003), 2.24 \pm 0.28$-fold $(p=0.001)$, and $1.55 \pm 0.06$-fold $(p=0.012)$, respectively (Fig. 9f). The immunofluorescence intensity of $\beta$-catenin in the hASC nucleus was also decreased in hASCs cotransfected with the let-7i-3p inhibitor and siLEF1 (Fig. 9g). Compared to those in the group transfected with the let$7 \mathrm{i}-3 \mathrm{p}$ inhibitor and siR-Ctrl, the protein expression levels of cytoplasmic and nuclear $\beta$-catenin were decreased $1.41 \pm 0.19$-fold $(p=0.036)$ and $2.13 \pm 0.35$-fold $(p=$ 


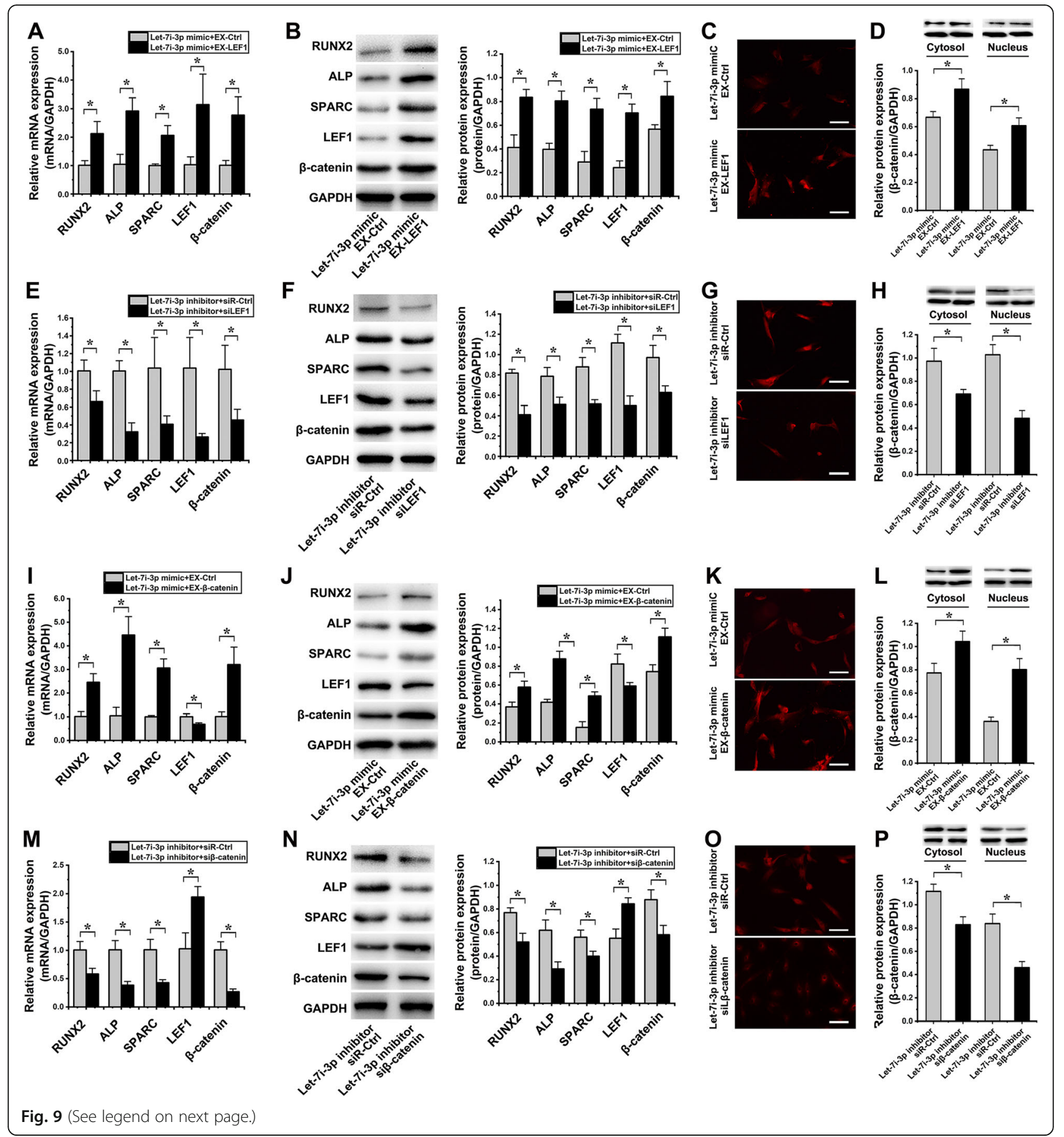

0.001), respectively, in hASCs cotransfected with the let7i-3p inhibitor and siLEF1 (Fig. 9h).

Compared to those in the group transfected with the let-7i-3p mimic and EX-Ctrl, the mRNA expression levels of RUNX2, ALP, SPARC, and $\beta$-catenin were increased $2.42 \pm 0.92$-fold $(p=0.004), 4.27 \pm 1.78$-fold $(p=$ $0.002), 3.06 \pm 0.41$-fold $(p=0.001)$, and $3.17 \pm 1.09$-fold $(p=0.008)$, the mRNA expression levels of LEF1 was decreased $1.47 \pm 0.21$-fold $(p=0.016)$, respectively, in
hASCs cotransfected with the let-7i-3p mimic and EX- $\beta$ catenin (Fig. 9i); the protein expression levels of the above markers were also increased: RUNX2, ALP, SPARC, and $\beta$-catenin were increased $1.57 \pm 0.06$-fold $(p=0.011), 2.10 \pm 0.03$-fold $(p=0.001), 3.16 \pm 1.01$-fold $(p=0.002)$, and $1.50 \pm 0.04$-fold $(p=0.006)$; the protein expression levels of LEF1 were decreased $1.40 \pm 0.13$-fold $(p=0.023)$, respectively (Fig. 9j). The immunofluorescence intensity of $\beta$-catenin in the hASC nucleus was 
(See figure on previous page.)

Fig. 9 Effect of LEF1 regulated by let-7i-3p on the osteogenic differentiation of hASCs under cyclic strain ${ }^{*} p<0.05$, significant differences existed between these two groups). a hASCs were cotransfected with the let-7i-3p mimic and EX-LEF1 under cyclic strain for 6 days, and the levels of the following mRNAs were significantly increased compared to those in hASCs cotransfected with let-7i-3p mimic and EX-Ctrl: RUNX2, ALP, SPARC, LEF1, and $\beta$-catenin ( $2.10 \pm 0.69$-fold, $p=0.014 ; 2.80 \pm 1.62$-fold, $p=0.005 ; 2.06 \pm 0.30$-fold, $p=0.006 ; 3.06 \pm 0.52$-fold, $p=0.029$; and 2.75 \pm 0.68 -fold, $p=0.010$, repectively). $\mathbf{b}$ Protein blots are listed in the left column. hASCs were cotransfected with the let-7i-3p mimic and EX-LEF1 under cyclic strain for 6 days. The protein expression levels of the above markers were also increased: RUNX (22.02 \pm 0.38 -fold, $p=0.004)$, ALP (2.03 \pm 0.07 -fold, $p=0.002)$, SPARC $(2.54 \pm 0.60$-fold, $p=0.004)$, LEF1 ( $2.91 \pm 0.61$-fold, $p=0.004)$, and $\beta$-catenin $(1.49 \pm 0.17$-fold, $p=0.022)$. c The immunofluorescence intensity of $\beta$-catenin in the nucleus was enhanced in hASCs cotransfected with the let-7i-3p mimic and EX-LEF1(scale bar $100 \mu \mathrm{m})$. $\mathbf{d}$ The protein expression levels of cytoplasmic and nuclear $\beta$-catenin in hASCs cotransfected with the let-7i-3p mimic and EX-LEF1 were respectively increased $1.30 \pm 0.10$-fold $(p=0.026)$ and $1.40 \pm 0.15$-fold $(p=0.017)$ than those in the group transfected with the let-7i-3p mimic and EX-Ctrl. e hASCs were cotransfected with the let-7i-3p inhibitor and siLEF1 under cyclic strain for 6 days. The mRNA levels of the above markers were decreased by $1.52 \pm 0.26$-fold $(p=0.026), 3.12 \pm 1.55$-fold $(p=0.002), 2.55 \pm 0.25$-fold $(p=0.038), 3.92 \pm 0.72$-fold $(p=0.018)$, and $2.25 \pm 1.24$ fold $(p=0.030)$, respectively, compared to those in hASCs cotransfected with the let-7i-3p inhibitor and siR-Ctrl. $\mathbf{f}$ Protein blots are listed in the left column. hASCs were cotransfected with the let-7i-3p inhibitor and siLEF1 under cyclic strain for 6 days. The protein expression levels of the above markers were also decreased $2.00 \pm 0.42$-fold $(p=0.002), 1.54 \pm 0.17$-fold $(p=0.013), 1.71 \pm 0.19$-fold $(p=0.003), 2.24 \pm 0.28$-fold $(p=0.001)$, and $1.55 \pm 0.06$-fold $(p=0.012)$, respectively. $\mathbf{g}$ The immunofluorescence intensity of $\beta$-catenin in the hASC nucleus was weakened in hASCs cotransfected with the let-7i-3p inhibitor and si $\beta$-catenin (scale bar $100 \mu \mathrm{m}$ ). $\mathbf{h}$ The protein expression levels of cytoplasmic and nuclear $\beta$-catenin in hASCs cotransfected with the let-7i-3p inhibitor and siLEF1were respectively decreased $1.41 \pm 0.19$-fold $(p=0.036)$ and $2.13 \pm 0.35$-fold $(p=$ 0.001) than those in the group transfected with the let-7i-3p inhibitor and siR-Ctrl. $\mathbf{i}$ hASCs were cotransfected with the let-7i-3p mimic and EX- $\beta$ catenin under cyclic strain for 6 days: compared to those in the group transfected with the let-7i-3p mimic and EX-Ctrl, the mRNA expression levels of RUNX2, ALP, SPARC, and $\beta$-catenin were increased $2.42 \pm 0.92$-fold $(p=0.004), 4.27 \pm 1.78$-fold $(p=0.002), 3.06 \pm 0.41$-fold $(p=0.001)$, and $3.17 \pm 1.09$-fold $(p=0.008)$; the mRNA expression levels of LEF1 was decreased $1.47 \pm 0.21$-fold $(p=0.016)$, respectively. $\mathbf{j}$ Protein blots are listed in the left column. hASCs were cotransfected with the let-7i-3p mimic and EX- $\beta$-catenin under cyclic strain for 6 days: compared to those in the group transfected with the let-7i-3p mimic and EX-Ctrl, the protein expression levels of RUNX2, ALP, SPARC and $\beta$-catenin were increased $1.57 \pm$ 0.06 -fold $(p=0.011), 2.10 \pm 0.03$-fold $(p=0.001), 3.16 \pm 1.01$-fold $(p=0.002)$, and $1.50 \pm 0.04$-fold $(p=0.006)$; the protein expression levels of LEF1 were decreased $1.40 \pm 0.13$-fold $(p=0.023$ ), respectively. $\mathbf{k}$ The immunofluorescence intensity of $\beta$-catenin in the hASC nucleus was enhanced in hASCs cotransfected with the let-7i-3p mimics and EX- $\beta$-catenin (scale bar $100 \mu \mathrm{m}$ ). I The protein expression levels of cytoplasmic and nuclear $\beta$ catenin in hASCs cotransfected with the let-7i-3p mimic and EX- $\beta$-catenin were respectively increased $1.35 \pm 0.12$-fold $(p=0.019)$ and $2.23 \pm 0.27$ fold $(p=0.008)$ than those in the group transfected with the let-7i-3p mimic and EX-Ctrl. $\mathbf{m}$ hASCs were cotransfected with the let-7i-3p mimic and EX- $\beta$-catenin under cyclic strain for 6 days: compared to those in the group transfected with the let-7i-3p inhibitor and si $\beta$-catenin, compared to those in the group transfected with the let-7i-3p inhibitor and siR-Ctrl, the mRNA levels of RUNX2, ALP, SPARC, and $\beta$-catenin were decreased $1.73 \pm 0.24$-fold $(p=0.015)$, $2.63 \pm 0.69$-fold $(p=0.004)$, $2.36 \pm 0.46$-fold $(p=0.006)$, and $3.75 \pm 0.21$-fold $(p=0.001)$; the mRNA expression levels of LEF1 was increased $1.89 \pm 0.74$-fold $(p=0.009$ ), respectively. $\mathbf{n}$ Protein blots are listed in the left column. hASCs were cotransfected with the let-7i-3p mimic and EX- $\beta$-catenin under cyclic strain for 6 days: compared to those in the group transfected with the let-7i-3p inhibitor and siR-Ctrl, the protein expression levels of RUNX2, ALP, SPARC, and $\beta$-catenin were decreased 1.48 \pm 0.15 -fold $(p=0.007), 2.12 \pm 0.14$-fold $(p=0.006$ ), $1.40 \pm 0.01$-fold $(p=0.020)$, and 1.51 \pm 0.17 -fold $(p=0.011)$; the protein expression levels of LEF1 were increased 1.53 \pm 0.19 -fold $(p=0.006)$, respectively. $\mathbf{o}$ The immunofluorescence intensity of $\beta$-catenin in the hASC nucleus was weaken in hASCs cotransfected with the let-7i-3p inhibitor and siß-catenin (scale bar $100 \mu \mathrm{m})$. p The protein expression levels of cytoplasmic and nuclear $\beta$-catenin in hASCs cotransfected with the let-7i-3p inhibitor and si $\beta$-catenin were respectively decreased $1.35 \pm 0.09$-fold $(p=0.006)$ and $1.83 \pm 0.04$-fold $(p=0.004)$ than those in the group transfected with the let-7i-3p inhibitor and siR-Ctrl

also increased in hASCs cotransfected with the let-7i-3p mimics and EX- $\beta$-catenin (Fig. 9k). Compared to those in the group transfected with the let-7i-3p mimic and EX-Ctrl, the protein expression levels of cytoplasmic and nuclear $\beta$-catenin were increased $1.35 \pm 0.12$-fold $(p=$ $0.019)$ and $2.23 \pm 0.27$-fold $(p=0.008)$, respectively, in hASCs cotransfected with the let-7i-3p mimic and EX- $\beta-$ catenin (Fig. 9l).

Compared to those in the group transfected with the let-7i-3p inhibitor and siR-Ctrl, the mRNA levels of RUNX2, ALP, SPARC, and $\beta$-catenin were decreased $1.73 \pm 0.24$-fold $(p=0.015), 2.63 \pm 0.69$-fold $(p=0.004)$, $2.36 \pm 0.46$-fold $(p=0.006)$, and $3.75 \pm 0.21$-fold $(p=$ $0.001)$; the mRNA expression levels of LEF1 was increased $1.89 \pm 0.74$-fold $(p=0.009)$, respectively, in hASCs cotransfected with the let-7i-3p inhibitor and si $\beta$-catenin (Fig. 9m); the protein expression levels of RUNX2, ALP, SPARC, and $\beta$-catenin were decreased
$1.48 \pm 0.15$-fold $(p=0.007), 2.12 \pm 0.14$-fold $(p=0.006)$, $1.40 \pm 0.01$-fold $(p=0.020)$, and $1.51 \pm 0.17$-fold $(p=$ 0.011 ); the protein expression levels of LEF1 were increased $1.53 \pm 0.19$-fold $(p=0.006)$, respectively (Fig. 9n). The immunofluorescence intensity of $\beta$-catenin in the hASC nucleus was also decreased in hASCs cotransfected with the let-7i-3p inhibitor and si $\beta$-catenin (Fig. 9o). Compared to those in the group transfected with the let-7i-3p inhibitor and siR-Ctrl, the protein expression levels of cytoplasmic and nuclear $\beta$-catenin were decreased $1.35 \pm 0.09$-fold $(p=0.006)$ and $1.83 \pm$ 0.04 -fold $(p=0.004)$, respectively, in hASCs cotransfected with the let-7i-3p inhibitor and si $\beta$-catenin (Fig. 9p).

Therefore, the potentiation of the let-7i-3p inhibitor on the osteogenic differentiation of hASCs could be weakened by LEF1 downregulation under cyclic strain. 


\section{Discussion}

Mechanical stimulation plays a vital role in bone formation and remolding and promotes angiogenesis. Bhatt et al. reported that a $3 \%$ cyclic strain increased the expression of OCN, OPN, and osteonectin in osteoblasts, and a 9\% strain further enhanced cell proliferation [26]. A study on cell lines showed that the expression of RUNX2 and COL-1 in BMSCs increased with a 0-9\% increase in tensile strain [27]. Some studies have shown that mechanical stimulation can promote the osteogenic differentiation and proliferation of bone MSCs and ASCs $[27,28]$. Li et al. reported that mechanical strain could inhibit the expression of PPAR $\gamma$ to impede differentiation into adipocytes [29]. However, mechanical stimulation, as an independent factor, could promote the efficiency of constructing tissue-engineered bone.

The Wnt signaling pathway regulates the process of osteogenic differentiation for MSCs and is involved in intramembranous and endochondral ossification [30]. Specific genes for osteogenic differentiation, such as RUNX2, Dlx5, and Osterix, can be upregulated by the activated Wnt/ $\beta$-catenin pathway, thereby promoting the osteogenic differentiation of BMSCs $[31,32]$. $\beta$-catenin can promote the progression of MSCs from osteoblastic precursor cells into more mature osteoblasts and can also suppress the differentiation of MSCs into adipogenic and chondrogenic lineages $[33,34]$. The Wnt/ $\beta$ catenin pathway inhibits the expression of PPAR $\gamma$ and CCAAT/enhancer-binding protein $\alpha$, the major adipogenic inducers, to inhibit adipogenic differentiation [35]. Additionally, mechanical stimulation can activate Wnt signaling pathways to promote the differentiation of stem cells into osteoblasts [36, 37]. Zhang et al. found that applying a static pressure of $100 \mathrm{kPa}$ to periodontal ligament stem cells activates the Wnt/ $\beta$-catenin pathway and regulates osteogenic differentiation [38]. Several studies have shown that tensile stress stimulation can also activate the $\mathrm{Wnt} / \beta$-catenin and $\mathrm{Wnt} / \mathrm{Ca}^{2+}$ pathways to promote the osteogenic differentiation of hASCs [39]. Evidence has shown that the Wnt signaling pathway regulates the osteogenic differentiation of MSCs [40]. In this study, we utilized cyclic strain to activate the Wnt/ $\beta$-catenin pathway and thus induce the osteogenic differentiation of hASCs. There might be some differences in the protocol for using $\mathrm{Wnt} / \beta$-catenin pathway activation to induce osteogenic differentiation compared to other methods, such as the conditioned medium, growth factors, and biomaterials. The mechanism by which the Wnt signaling pathway is activated by mechanical stimulation and the difference between using mechanical and chemical stimulation to activate this signaling pathway are still not understood.

LEF1 is an essential member of the effector protein family TCF/LEF that acts at the end of the Wnt signaling pathway. This protein forms a dimer with excessively aggregated $\beta$-catenin in the activated Wnt signaling pathway in the cytoplasm. Then, these complexes enter the nucleus to promote gene transcription and thus play regulatory roles in signaling. LEF1 plays a crucial regulatory role in the development of bone [41]. Hoeppner et al. reported that LEF1 $\mathrm{n}$ binds $\beta$-catenin, stimulates LEF/TCF reporter activity, and promotes terminal osteoblast differentiation [42]. LEF1 transcription factors play essential roles in proper osteogenesis and bone volume loss in $\mathrm{LEF} 1^{+/-}$mice in the early years of life [43]. In the present study, we reduced the expression of LEF1 and significantly inhibited the osteogenic differentiation of hASCs. This result is generally consistent with the above findings.

Let-7i-3p, a type of microRNA (miRNA), is involved in many different types of diseases. Falzone et al. reported that abnormal let-7i-3p expression has the potential to predict recurrence in oral cancer patients [44]. Indersie et al. found that let-7i-3p expression was decreased in pediatric hepatoblastoma and inhibited hepatoblastoma cell growth and Wnt signaling activity in vitro partly through $\beta$-catenin downregulation [45]. In type 2 diabetes patients, the urinary extracellular vesicle miRNA signature had increased levels of let-7i-3p, which is a noninvasive early biomarker of diabetic nephropathy in patients with type 2 diabetes with the "Asian Indian phenotype" [46]. Gu et al. reported that let-7i-3p was significantly overexpressed in the serum of mandibular prognathism patients, indicating that let-7i-3p might be associated with bone development and growth [47]. Mandibular prognathism is characterized by bone overdevelopment and growth, which appears to be inconsistent with our findings. We found that overexpression of let-7i-3p could inhibit the osteogenic differentiation of hASCs. The reasons for this seemingly opposite result need to be studied further. Thus far, we are unaware of any study on the expression of let-7i-3p affecting bone formation, and this study is the first report on this issue.

Studies have been conducted to directly regulate the expression of LEF1 by miRNAs, mainly focusing on tumor diseases. miR-34a increased chemosensitivity in BIU87/ADR cells by targeted inhibition of the TCF1/ LEF1 axis [48]. miR-22, a direct target of H19, binds to the 3'UTR of LEF1 to inhibit its expression and reverse the effect of $\mathrm{H} 19$ on nucleus pulposus cells, thereby inhibiting the Wnt/ $\beta$-catenin pathway [49]. Overexpression of miR-708 inhibits the proliferation, invasion, migration, and epithelial-mesenchymal transition but also promotes the apoptosis of melanoma cells by targeting LEF1 through suppression of the Wnt signaling pathway [50]. The expression of miR219-5p was shown to be decreased in colorectal cancer and to inhibit colorectal cancer metastasis and 
epithelial-mesenchymal transition by targeting LEF1 to inactivate the AKT and ERK pathways [51]. Suppressed miR-557 negatively regulates the expression of LEF1 to inhibit the proliferation of lung cancer cells [52].

Currently, some studies have confirmed that some miRNAs can affect the expression of LEF1 and the $\mathrm{Wnt} / \beta$-catenin pathway in the process of osteogenesis. Overexpression of miR-539 increased the expression of $\beta$-catenin, LEF1, c-myc, cyclin D1, RUNX2, BGP, and BMP-2 in rat osteoblasts [53]. Overexpression of miR-10a inhibited the osteogenic differentiation of MC3T3-E1 cells by inactivating the Wnt signaling pathway; the expression of LEF1 was also decreased during this process [54]. However, reports of miRNAs directly regulating LEF1 and affecting osteogenesis have not been published. This study is the first to investigate the direct regulation of LEF1 expression by miRNAs and show that a miRNA affects the osteogenic differentiation of hASCs.

\section{Conclusion}

To determine the role of let-7i-3p in the process of hASC osteogenic differentiation under cyclic strain, we designed serial experiments to verify its function. Inhibition of let-7i-3p and overexpression of LEF1 promoted

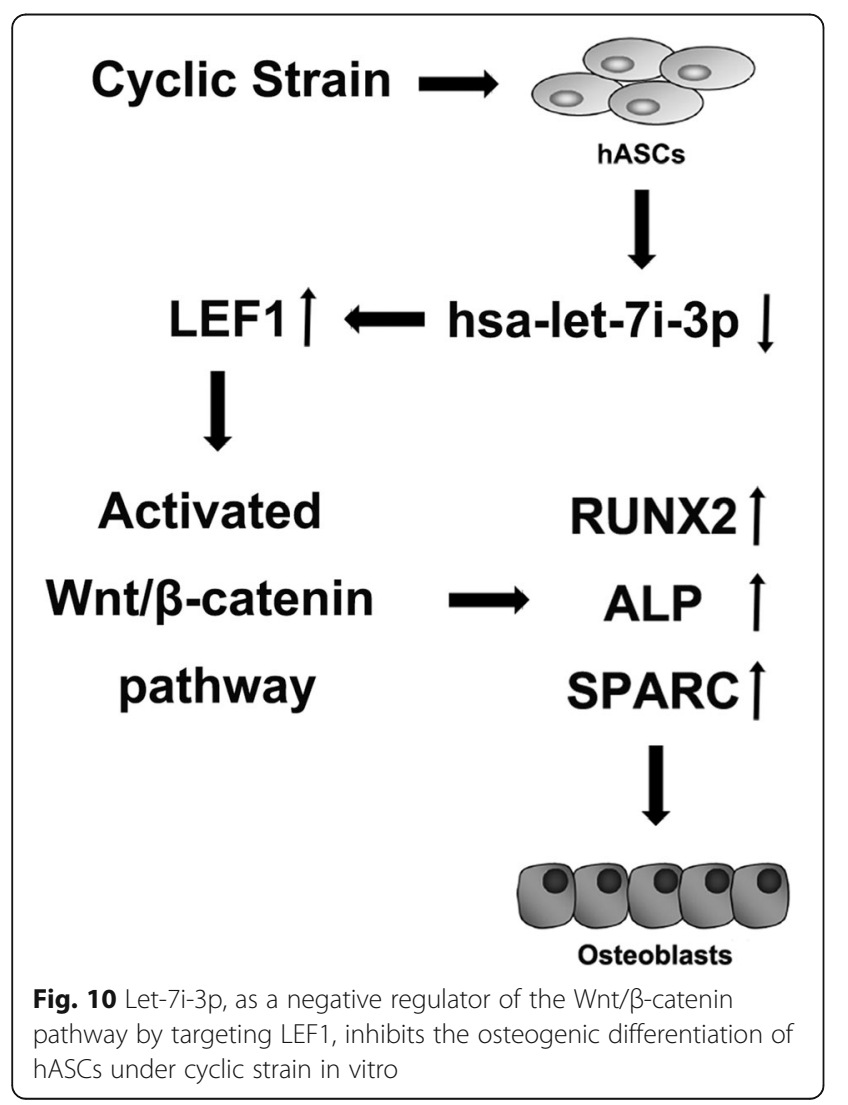

the osteogenic differentiation of hASCs in vitro. Furthermore, gain- and loss-of-function experiments on let-7i$3 p$ showed that let-7i-3p negatively regulates the $\mathrm{Wnt} / \beta$ catenin pathway via LEF1, which inhibits the osteogenic differentiation of hASCs under cyclic strain in vitro (Fig. 10).

\section{Supplementary information}

Supplementary information accompanies this paper at https://doi.org/10. 1186/s13287-019-1470-z.

Additional file 1. The sequences mentioned in the study

Additional file 2: Figure S1. Microarray for miRNA changes under cyclic strain. There were 150 miRNAs decreased and 87 miRNAs increased significantly (>2-fold).

\section{Abbreviations}

hASCs: Human adipose-derived stem cells; miRNAs: MicroRNAs;

LEF1: Lymphoid enhancer factor 1; BMSCs: Bone marrow mesenchymal stem cells; PBS: Phosphate-buffered saline; a-MEM: Alpha-modified Eagle's medium; FBS: Fetal bovine serum; CCK-8: Cell Counting Kit-8 assay; qPCR: Real-time quantitative PCR; SDS: Sodium dodecyl sulfate; EX-LEF1: LEF1 expression plasmid; siLEF1: LEF1 siRNA; EX-Ctrl: Negative control expression plasmid; siR-Ctrl: Negative control siRNA; miR-Ctrl: Mimic negative control of the let-7i-3p mimic; miR-Ctrl: Inhibitor negative control of the let-7i-3p inhibitor; wt-LEF1: LEF1 3'UTR-wild type; mu-LEF1: LEF1 3'UTR-mutant;

ALP: Alkaline phosphatase; RUNX2: Runt-related transcription factor 2; SPARC: Secreted protein acidic and cysteine-rich; GAPDH: Glyceraldehyde-3phosphate dehydrogenase; ANOVA: Analysis of variance

Acknowledgements

All authors are acknowledged for their contribution to the study.

\section{Authors' contributions}

$H D, R G, X D, H W$, and $Y L$ were in charge of the conception, study design, and literature research. $Y L, M L, Y M, H J$, and $H S$ were in charge of the experimental studies. SL, XD, and CW were in charge of the data analysis/ interpretation. $H D, Y L, R G$, and $H W$ were in charge of the manuscript preparation, editing, revision, and approval of the final version. All authors read and approved the final version of the manuscript.

Authors' information

Not applicable.

\section{Funding}

Our work was funded by the National Natural Science Foundation of China (grant agreement number 81600908).

\section{Availability of data and materials}

The datasets generated and/or analyzed during the current study are not publicly available due but are available from the corresponding author upon reasonable request.

Ethics approval and consent to participate

Fat tissue donors signed informed consent. This study was approved by the ethics committee of the Nanjing Medical University.

Consent for publication

Not applicable.

\section{Competing interests}

The authors declare that they have no competing interests.

\section{Author details}

${ }^{1}$ Department of Oral and Maxillofacial Surgery, Affiliated Stomatological Hospital of Nanjing Medical University, Hanzhong Road No.136, Nanjing 210029, Jiangsu Province, People's Republic of China. ${ }^{2}$ Oral Disease Key 
Laboratory of Jiangsu Province, Nanjing Medical University, Nanjing 210029, Jiangsu Province, People's Republic of China. ${ }^{3}$ Department of Nuclear Medicine, Fujian Medical University Union Hospital, Fuzhou 350001, Fujian Province, People's Republic of China.

\section{Received: 29 July 2019 Revised: 16 October 2019}

\section{Accepted: 23 October 2019 Published online: 21 November 2019}

\section{References}

1. Wang ZX, Chen C, Zhou Q, Wang XS, Zhou G, Liu W, et al. The treatment efficacy of bone tissue engineering strategy for repairing segmental bone defects under osteoporotic conditions. Tissue Eng Part A. 2015;21:2346-55.

2. Seitz H, Rieder W, Irsen S, Leukers B, Tille C. Three-dimensional printing of porous ceramic scaffolds for bone tissue engineering. J Biomed Mater Res B Appl Biomater. 2005;74:782-8.

3. Jin Q, Yuan K, Lin W, Niu C, Ma R, Huang Z. Comparative characterization of mesenchymal stem cells from human dental pulp and adipose tissue for bone regeneration potential. Artif Cells Nanomed Biotechnol. 2019; 47:1577-84.

4. Polymeri A, Giannobile W, Kaigler D. Bone marrow stromal stem cells in tissue engineering and regenerative medicine. Horm Metab Res. 2016;48: $700-13$

5. Li M, Li X, Meikle MC, Islam I, Cao T. Short periods of cyclic mechanical strain enhance triple-supplement directed osteogenesis and bone nodule formation by human embryonic stem cells in vitro. Tissue Eng Part A. 2013; 19:2130-7.

6. Zuk PA, Zhu M, Ashjian P, De Ugarte DA, Huang Jl, Mizuno H, et al. Human adipose tissue is a source of multipotent stem cells. Mol Biol Cell. 2002;13: 4279-95.

7. Huang SJ, Fu RH, Shyu WC, Liu SP, Jong GP, Chiu YW, et al. Adipose-derived stem cells: isolation, characterization, and differentiation potential. Cell Transplant. 2013;22:701-9.

8. Vidal MA, Walker NJ, Napoli E, Borjesson DL. Evaluation of senescence in mesenchymal stem cells isolated from equine bone marrow, adipose tissue, and umbilical cord tissue. Stem Cells Dev. 2012;21:273-83.

9. Liao HT, Chen CT. Osteogenic potential: comparison between bone marrow and adipose-derived mesenchymal stem cells. World J Stem Cells. 2014;6: 288-95.

10. Chen S, Zheng Y, Zhang S, Jia L, Zou Y. Promotion effects of miR-375 on the osteogenic differentiation of human adipose-derived mesenchymal stem cells. Stem Cell Rep. 2017;8:773-86.

11. Fan C, Jia L, Zheng Y, Jin C, Liu Y, Liu H, et al. MiR-34a promotes osteogenic differentiation of human adipose-derived stem cells via the RBP2/NOTCH1/ CYCLIN D1 coregulatory network. Stem Cell Rep. 2016;7:236-48.

12. Choi JR, Yong KW, Choi JY. Effects of mechanical loading on human mesenchymal stem cells for cartilage tissue engineering. J Cell Physiol. 2018; 233:1913-28.

13. Nam HY, Pingguan-Murphy B, Abbas AA, Merican AM, Kamarul T. Uniaxial cyclic tensile stretching at $8 \%$ strain exclusively promotes tenogenic differentiation of human bone marrow-derived mesenchymal stromal cells. Stem Cells Int. 2019;2019:9723025.

14. Pinzone JJ, Hall BM, Thudi NK, Vonau M, Qiang YW, Rosol TJ, et al. The role of Dickkopf-1 in bone development, homeostasis, and disease. Blood. 2009; 113:517-25.

15. Balemans W, Van Hul W. The genetics of low-density lipoprotein receptorrelated protein 5 in bone: a story of extremes. Endocrinology. 2007;148: 2622-9.

16. Zheng HF, Tobias JH, Duncan E, Evans DM, Eriksson J, Paternoster L, et al. WNT16 influences bone mineral density, cortical bone thickness, bone strength, and osteoporotic fracture risk. PLoS Genet. 2012;8:e1002745.

17. Shahi M, Peymani A, Sahmani M. Regulation of bone metabolism. Rep Biochem Mol Biol. 2017;5:73-82

18. Zhao XE, Yang Z, Gao Z, Ge J, Wei Q, Ma B. 6-Bromoindirubin-3'-oxime promotes osteogenic differentiation of canine BMSCs through inhibition of GSK3beta activity and activation of the Wnt/beta-catenin signaling pathway. An Acad Bras Cienc. 2019:91:e20180459.

19. Baron R, Kneissel M. WNT signaling in bone homeostasis and disease: from human mutations to treatments. Nat Med. 2013;19:179-92.

20. Benham-Pyle BW, Sim JY, Hart KC, Pruitt BL, Nelson WJ. Increasing betacatenin/Wnt3A activity levels drive mechanical strain-induced cell cycle progression through mitosis. eLife. 2016;5:e19799.
21. Yan $Y$, Sun H, Gong Y, Yan Z, Zhang X, Guo Y, et al. Mechanical strain promotes osteoblastic differentiation through integrin-beta1-mediated betacatenin signaling. Int J Mol Med. 2016;38:594-600.

22. Liu B, Li J, Cairns MJ. Identifying miRNAs, targets and functions. Brief Bioinform. 2014;15:1-19.

23. Zhang J, Tu Q, Bonewald LF, He X, Stein G, Lian J. Effects of miR-335-5p in modulating osteogenic differentiation by specifically downregulating Wnt antagonist DKK1. J Bone Miner Res. 2011;26:1953-63.

24. Eskildsen T, Taipaleenmaki H, Stenvang J, Abdallah BM, Ditzel N, Nossent AY, et al. MicroRNA-138 regulates osteogenic differentiation of human stromal (mesenchymal) stem cells in vivo. Proc Natl Acad Sci U S A. 2011;108:6139-44.

25. Wang J, Guan X, Guo F, Zhou J, Chang A, Sun B, et al. miR-30e reciprocally regulates the differentiation of adipocytes and osteoblasts by directly targeting low-density lipoprotein receptor-related protein 6. Cell Death Dis. 2013;4:e845

26. Bhatt KA, Chang El, Warren SM, Lin SE, Bastidas N, Ghali S, et al. Uniaxial mechanical strain: an in vitro correlate to distraction osteogenesis. J Surg Res. 2007;143:329-36.

27. Jiang Y, Wang Y, Tang G. Cyclic tensile strain promotes the osteogenic differentiation of a bone marrow stromal cell and vascular endothelial cell co-culture system. Arch Biochem Biophys. 2016;607:37-43.

28. Hanson AD, Marvel SW, Bernacki SH, Banes AJ, van Aalst J, Balasubramaniam EG. Osteogenic effects of rest inserted and continuous cyclic tensile strain on hASC lines with disparate osteodifferentiation capabilities. Ann Biomed Eng. 2009;37:955-65.

29. Li R, Liang L, Dou Y, Huang Z, Mo H, Wang Y, et al. Mechanical strain regulates osteogenic and adipogenic differentiation of bone marrow mesenchymal stem cells. Biomed Res Int. 2015;2015:873251.

30. Wang J, Wang CD, Zhang N, Tong WX, Zhang YF, Shan SZ, et al. Mechanical stimulation orchestrates the osteogenic differentiation of human bone marrow stromal cells by regulating HDAC1. Cell Death Dis. 2016;7:e2221.

31. Jiang Z, Von den Hoff JW, Torensma R, Meng L, Bian Z. Wnt16 is involved in intramembranous ossification and suppresses osteoblast differentiation through the Wnt/beta-catenin pathway. J Cell Physiol. 2014:229:384-92.

32. Chen $H$, Mo D, Li M, Zhang $Y$, Chen L, Zhang $X$, et al. miR-709 inhibits 3T3L1 cell differentiation by targeting GSK3beta of Wnt/beta-catenin signaling. Cell Signal. 2014;26:2583-9.

33. Wu L, Wei $Q, L v Y$, Xue J, Zhang B, Sun $Q$, et al. Wnt/beta-catenin pathway is involved in cadmium-induced inhibition of osteoblast differentiation of bone marrow mesenchymal stem cells. Int J Mol Sci. 2019;110(3):545-53.

34. Case N, Rubin J. Beta-catenin--a supporting role in the skeleton. J Cell Biochem. 2010;110:545-53.

35. Kang S, Bennett CN, Gerin I, Rapp LA, Hankenson KD, Macdougald OA. Wnt signaling stimulates osteoblastogenesis of mesenchymal precursors by suppressing CCAAT/enhancer-binding protein alpha and peroxisome proliferator-activated receptor gamma. J Biol Chem. 2007;282:14515-24.

36. Luan F, Ma K, Mao J, Yang F, Zhang M, Luan H. Differentiation of human amniotic epithelial cells into osteoblasts is induced by mechanical stretch via the Wnt/betacatenin signalling pathway. Mol Med Rep. 2018;18:5717-25.

37. Sindhavajiva PR, Sastravaha P, Arksornnukit M, Pavasant P. Intermittent compressive force induces human mandibular-derived osteoblast differentiation via WNT/beta-catenin signaling. J Cell Biochem. 2018;119. 3474-85.

38. Zhang L, Liu W, Zhao J, Ma X, Shen L, Zhang Y, et al. Mechanical stress regulates osteogenic differentiation and RANKL/OPG ratio in periodontal ligament stem cells by the Wnt/beta-catenin pathway. Biochim Biophys Acta. 1860;2016:2211-9.

39. Du HM, Zheng XH, Wang LY, Tang $W$, Liu L, Jing $W$, et al. The osteogenic response of undifferentiated human adipose-derived stem cells under mechanical stimulation. Cells Tissues Organs. 2012;196:313-24.

40. Zhang R, Oyajobi BO, Harris SE, Chen D, Tsao C, Deng HW, et al. Wnt/betacatenin signaling activates bone morphogenetic protein 2 expression in osteoblasts. Bone. 2013;52:145-56.

41. Ma B, Hottiger MO. Crosstalk between Wnt/beta-catenin and NF-kappaB signaling pathway during inflammation. Front Immunol. 2016;7:378

42. Hoeppner LH, Secreto FJ, Razidlo DF, Whitney TJ, Westendorf JJ. Lef1DeltaN binds beta-catenin and increases osteoblast activity and trabecular bone mass. J Biol Chem. 2011:286:10950-9.

43. Noh T, Gabet Y, Cogan J, Shi Y, Tank A, Sasaki T, et al. Lef1 haploinsufficient mice display a low turnover and low bone mass phenotype in a genderand age-specific manner. PLoS One. 2009;4:e5438. 
44. Falzone L, Lupo G, Rosa GRM, Crimi S, Anfuso CD, Salemi R, et al. Identification of novel microRNAs and their diagnostic and prognostic significance in oral cancer. Cancers (Basel). 2019;11(5):610.

45. Indersie E, Lesjean S, Hooks KB, Sagliocco F, Ernault T, Cairo S, et al. MicroRNA therapy inhibits hepatoblastoma growth in vivo by targeting beta-catenin and Wnt signaling. Hepatol Commun. 2017;1:168-83.

46. Prabu P, Rome S, Sathishkumar C, Gastebois C, Meugnier E, Mohan V, et al. MicroRNAs from urinary extracellular vesicles are non-invasive early biomarkers of diabetic nephropathy in type 2 diabetes patients with the 'Asian Indian phenotype'. Diabetes Metab. 2019;45:276-85.

47. Gu Y, Zhang Y, Zhao C, Pan Y, Smales RJ, Wang H, et al. Serum microRNAs as potential biomarkers of mandibular prognathism. Oral Dis. 2014;20:55-61.

48. Liu X, Liu X, Wu Y, Fang Z, Wu Q, Wu C, et al. MicroRNA-34a attenuates metastasis and chemoresistance of bladder cancer cells by targeting the TCF1/LEF1 Axis. Cell Physiol Biochem. 2018:48:87-98.

49. Wang $X$, Zou M, Li J, Wang B, Zhang Q, Liu F, et al. LncRNA H19 targets miR-22 to modulate $\mathrm{H} 2 \mathrm{O} 2$-induced deregulation in nucleus pulposus cell senescence, proliferation, and ECM synthesis through Wnt signaling. J Cell Biochem. 2018;119:4990-5002.

50. Song XF, Wang QH, Huo R. Effects of microRNA-708 on epithelialmesenchymal transition, cell proliferation and apoptosis in melanoma cells by targeting LEF1 through the Wnt signaling pathway. Pathol Oncol Res. 2019;25:377-89

51. Huang $L X$, Hu CY, Jing L, Wang MC, Xu M, Wang J, et al. microRNA-219-5p inhibits epithelial-mesenchymal transition and metastasis of colorectal cancer by targeting lymphoid enhancer-binding factor 1. Cancer Sci. 2017; 108:1985-95.

52. Qiu J, Hao Y, Huang S, Ma Y, Li X, Li D, et al. MiR-557 works as a tumor suppressor in human lung cancers by negatively regulating LEF1 expression. Tumour Biol. 2017;39:1010428317709467.

53. Zhu XB, Lin WJ, Lv C, Wang L, Huang ZX, Yang SW, et al. MicroRNA-539 promotes osteoblast proliferation and differentiation and osteoclast apoptosis through the AXNA-dependent Wnt signaling pathway in osteoporotic rats. J Cell Biochem. 2018;119:8346-58.

54. Li J, Zhang Y, Zhao Q, Wang J, He X. MicroRNA-10a influences osteoblast differentiation and angiogenesis by regulating beta-catenin expression. Cell Physiol Biochem. 2015;37:2194-208.

\section{Publisher's Note}

Springer Nature remains neutral with regard to jurisdictional claims in published maps and institutional affiliations.

Ready to submit your research? Choose BMC and benefit from:

- fast, convenient online submission

- thorough peer review by experienced researchers in your field

- rapid publication on acceptance

- support for research data, including large and complex data types

- gold Open Access which fosters wider collaboration and increased citations

- maximum visibility for your research: over $100 \mathrm{M}$ website views per year

At $\mathrm{BMC}$, research is always in progress.

Learn more biomedcentral.com/submissions 